\title{
APPORT DE L'IMAGERIE ALSAT2 POUR LA MAINTENANCE DU CADASTRE STEPPIQUE EN ALGERIE
}

Mohamed Akram SEDDIKI 1,2,3 (Chargé de recherche), Sid Ahmed SOUIAH ${ }^{1,2}$ (Maitre de Conférence A), Tarek GHODBANI ${ }^{1,2}$ (Professeur, Directeur du Laboratoire EGEAT), Abd El Halim GUERROUDJ 4 (Assistant d'études A), Fatiha ZERGUI ${ }^{3}$ (Ingénieur d'état), Mohamed MILOUA ${ }^{5}$ (Ingénieur d'état). Akramcnts@yahoo.fr ou aseddiki@cts.asal.dz

N01, Avenue de la Palestine, BP13, Arzew 31200, Oran, Algérie, Télé : +21341793042, Fax $:+21341792176$

1 : Faculté des Sciences de la terre et de l'Univers, Département de Géographie, Université Oran2, Algérie

2 : Laboratoire EGEAT Espace Géographique et Aménagement du Territoire, Université Oran2, Algérie

3 : Centre des Techniques Spatiales/Agence Spatiale Algérienne, Algérie

4 : Université Ammar THLIDJI, Laghouat, Algérie

Résumé

5 : Institut National de Cartographie et de Télédétection INCT, Algérie

L'établissement du cadastre général en Algérie et la constitution du livre foncier ont été entrepris dès 1977. Cette opération a concerné, en premier lieu, les régions nord du territoire national (les zones rurales et les zones urbaines) et les agglomérations des régions sud.

Ce projet porte sur un vaste territoire couvrant les zones dites 'steppiques' et s'inscrit dans le cadre de recherche de solutions techniques relatives à l'exploitation de l'imagerie du satellite ALSAT2 pour la maintenance des bases de données graphiques du cadastre steppique. Cela par la détermination des capacités informatives que possède cette image en matière d'identification des objets correspondant à une partie ou la totalité des propriétés foncières. Les informations extraites feront partie intégrante d'un Système d'Informations Foncières multi-sources SIF (données du cadastre, d'urbanisme et base de données ortho-image).

Mots clés : Imagerie ALSAT2, Algérie, Cadastre Steppique, SIF, classification K-Means, ISODATA, Orientée objet.

\section{Abstract}

The establishment of the general cadastre in Algeria and the constitution of the land register were undertaken, in 1977 and first of all, in the northern regions of the national territory, in rural and urban areas, as well as in the urban areas of the southern regions.

This project covers a vast territory covering the so-called 'steppe' areas and is part of a research framework for technical solutions relating to the exploitation of the imagery of the ALSAT2 satellite for the maintenance of the graphic databases of the steppe land register, this, by determining the informative capacities that this image possesses in terms of identifying objects corresponding to part or all of the land holdings. The information extracted will be an integral part of a multi-source Land Information System SIF (cadastral data, planning data and ortho-image database).

Keywords: ALSAT2 Imagery, Algeria, Steppique Cadster, SIF, K-Means classification, ISODATA, Oriented Object.

\section{Introduction}

En Algérie, comme défini dans la loi $n^{\circ} 90-25$ du $18 / 11 / 1990$ portant orientation foncière, une terre saharienne est toute terre située en-dessous de l'isohyète de $100 \mathrm{~mm}$. Les terres steppiques sont constituées, au sens de cette même loi, par des terres à vocation pastorale ou alfatière (figure 01 ). Compte tenu de l'étendue des superficies des zones steppiques et de la nature des territoires à traiter, qui constituent dans leur quasi-totalité des zones ayant un statut de propriété publique, les procédés techniques choisis pour la réalisation du cadastre des zones nord du territoire national (délimitation des propriétés basée sur les orthophotoplans et les plans de restitution) sont devenus inadaptés.

Cependant, la délimitation des propriétés en zones steppiques s'effectue à travers des travaux de bureau en se basant sur des documents cartographiques (généralement la couverture cartographique existante est à petite échelle) ce qui donne lieu à la confection d'une matrice cadastrale. Cette dernière comporte la liste des propriétés de l'Etat et un plan cadastral pour constituer le Livre Foncier au profit de l'Etat (des titres de propriété sous forme de livrets fonciers). Ce genre de délimitation au bureau par des supports cartographiques (sans recourir à des images satellitaires ni à une enquête sur le terrain) présente un défaut d'omission des propriétés privées et des terres 'Arch ' [terres dont les tribus jouissaient en permanence, collectivement, suivant les besoins de la communauté. Gérées par un conseil composé de notables de la tribu, elles étaient juridiquement enregistrées au compte de l'Etat depuis la réforme agraire en 1972.] (R. Bensouiah, 2003 et A Ali, 2011).

Ce projet s'inscrit dans un cadre de recherche de solutions techniques relatives à l'exploitation de l'imagerie du satellite ALSAT2 (construit dans le cadre d'un partenariat entre l'ASAL-Algérie et EADS AstriumFrance (Herbert J. Kramer, 2002) pour la maintenance du cadastre steppique. Cela par la détermination des capacités informatives que possède cette image en matière d'identification des objets correspondant à une partie ou la totalité des propriétés foncières. Cette question d'identification, à partir des images à haute et très haute résolution spatiale, a été abordée par 
plusieurs auteurs et consiste à explorer son contenu et sa précision (M. Ettarid, 2004, S. L-Homme, 2005) ; en précisant en outre la détermination de l'échelle géométrique, la base de données des objets interprétés et sa correspondance ou non aux différentes échelles (D. Holland, 2006).

La comparaison des différentes méthodes d'extraction de l'information par classification à partir des images spatiales et la réduction du taux d'incertitude (A. Masse, 2013), permettent aux services du cadastre (par l'utilisation des capacités du satellite ALSAT2) de mettre à jour les bases de données graphiques géoréférencées après un recensement précis des îlots à l'échelle des sections cadastrales et des communes (enregistrés juridiquement au nom de l'état alors qu'ils appartiennent réellement à des propriétaires privés, des usufruitiers ou des occupants des terres Arch.

Les premières solutions testées dans le cadre de ce travail avaient pour base l'utilisation des algorithmes de classification standards qui sont adaptées aux parcelles de taille grande ou moyennes par rapport à la résolution spatiale de l'image support d'étude. Pour les îlots composés de bâtiments isolés de petites dimensions, une approche d'apprentissage a été développée.

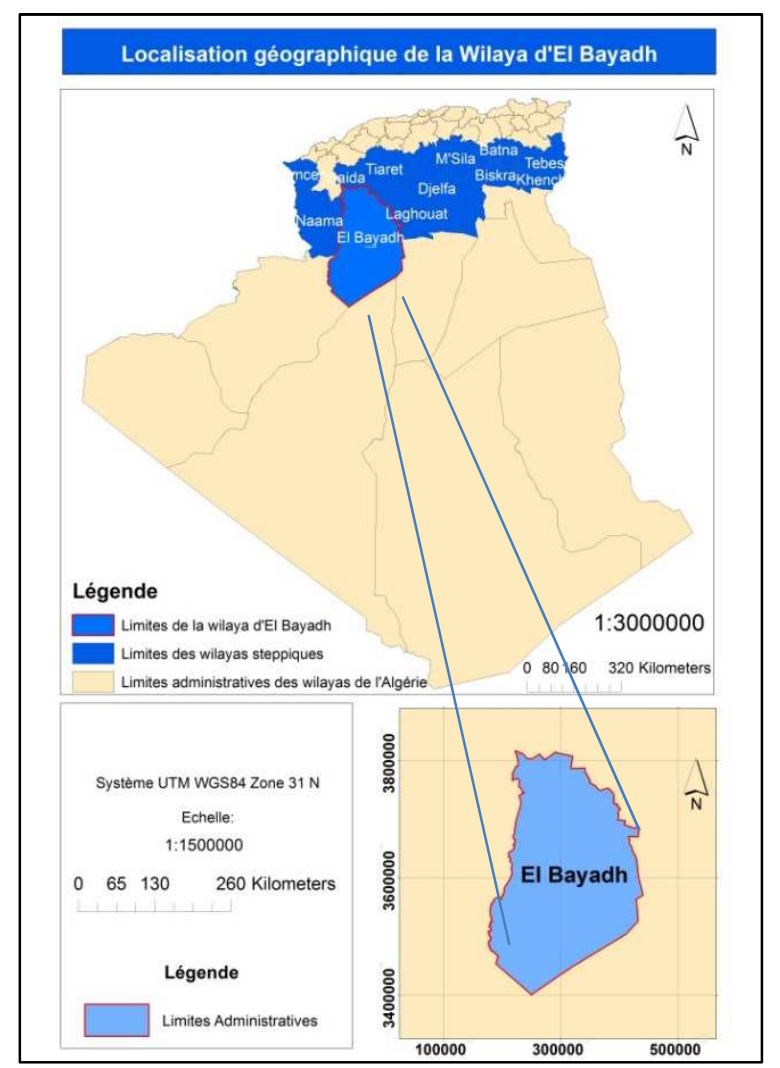

Figure 1 : Localisation du territoire steppique sur la carte de l'Algérie (14\% du territoire national).

\section{Données et zone d'étude}

La wilaya d'El Bayadh fait partie intégrante de la région des Hautes Plaines steppiques du Sud-Ouest algérien. Sur le plan physique, elle présente trois grandes zones distinctes :

- Au nord : les Hautes Plaines :

- Au centre : l'Atlas saharien ;
- Au sud : la frontière présaharienne.

Dans notre étude, pour des raisons de disponibilité des données, on a choisi deux communes : La commune d'El Bayadh d'une superficie de $48783 \mathrm{Ha}$ et la commune de Rogassa qui couvre environ $241600 \mathrm{Ha}$, délimitées géographiquement par les parallèles $33^{\circ}, 26$ et $34^{\circ}, 16$ Nord, et les méridiens $0^{\circ}, 73$ et $1^{\circ}, 23$ Est.

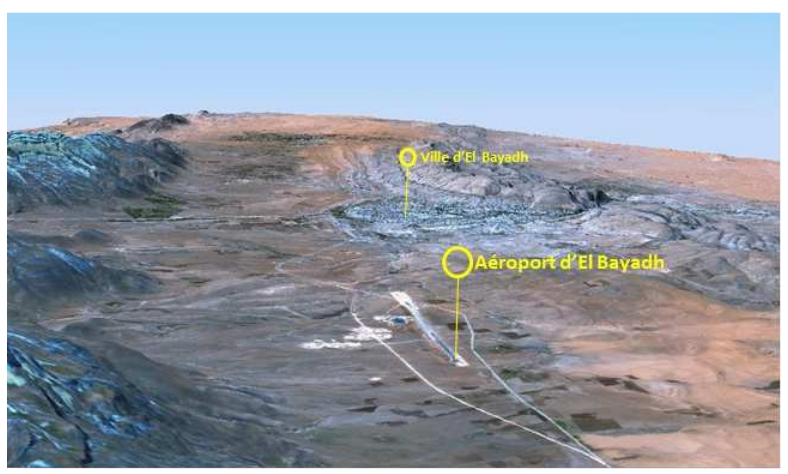

Figure 2 : Ortho-image AISAT2 drapée sur le MNT de la ville d'El Bayadh (altitude moyenne de $1313 \mathrm{~m}$ ).

Concernant les données images, sur un nombre de onze (11) scènes, qui couvrent la zone d'étude, sept (07) images ont été utilisées pour produire le support orthoimage dont une scène utilisée pour la détermination de l'échelle par étude du potentiel d'identification des objets. Il s'agit d'une évaluation du potentiel cartographique de l'imagerie satellite qui doit se faire sur la base de critères permettant de conférer au produit les qualités suivantes: - La possibilité de pouvoir identifier sans ambiguïté, sur l'image, le type et la nature des objets à représenter sur une carte à une échelle donnée. - La précision de la localisation de ces objets identifiés. (M. Ettarid, 2004).

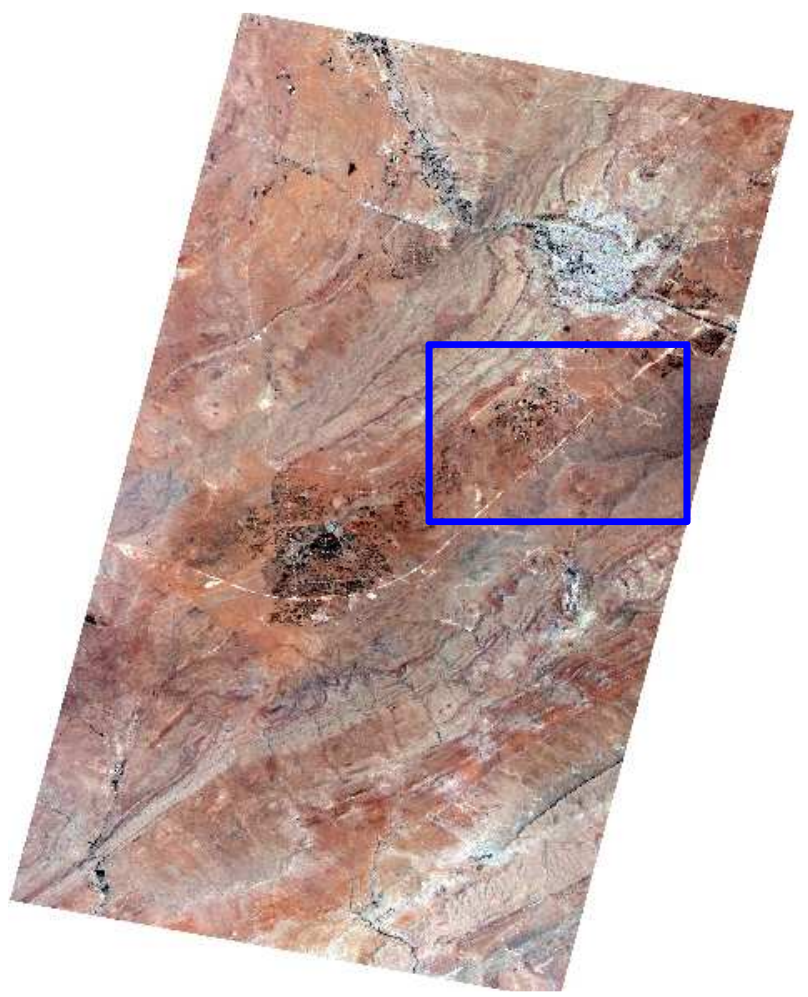

Revue Française de Photogrammétrie et Télédétection Volume 223 (année 2021), numéro Spécial Afrique https://doi.org/10.52638/rfpt.2021.504 
Figure 3 : Exemple d'un segment composé de deux images ALSAT2.

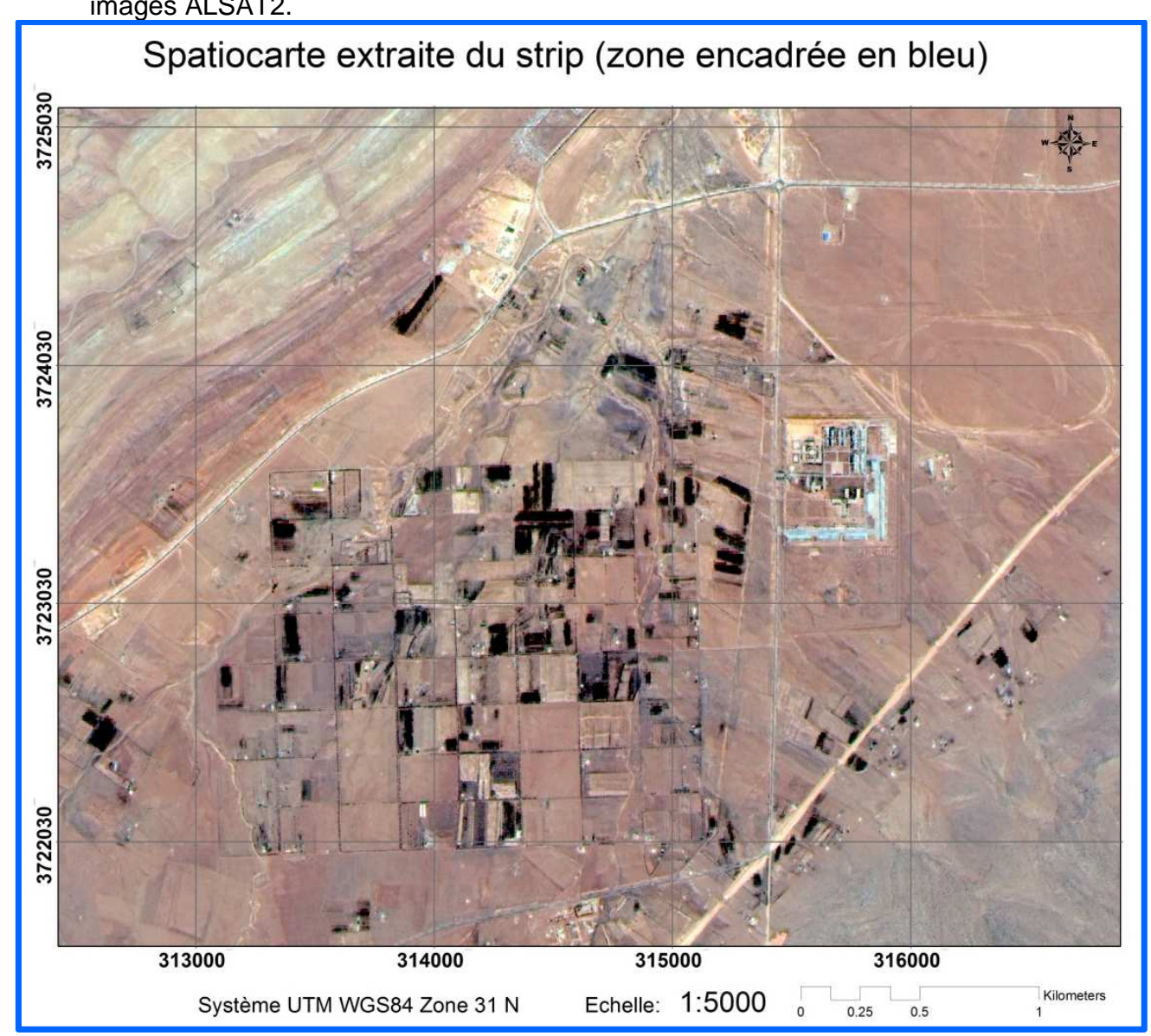

Figure 4 : Zoom sur la partie encadrée en bleu sur le segment ALSAT2 (figure 3).

\begin{tabular}{|c|c|}
\hline Image ALSAT2 & Caractéristiques \\
\hline Nom de l'image & A1M 2017-06-22 09:58:23.6338960 \\
\hline $\begin{array}{l}\text { Dimension de la scène (lignes et } \\
\text { colonnes) }\end{array}$ & $1750 \times 1750$ \\
\hline Format de stockage & GEOTIFF \\
\hline Date de prise de vue & $22-06-2017$ \\
\hline Heure de prise de vue & 09 h 58 '23”' \\
\hline $\begin{array}{l}\text { Système de coordonnées } \\
\text { géographiques }\end{array}$ & WGS 84 \\
\hline Nombre de bandes spectrales & 4 Bandes \\
\hline Résolution spatiale (mètre) & $2,5 \times 2,5$ \\
\hline Localisation géographique & $\begin{array}{lc}\lambda \min =00^{\circ} 51^{\prime} 40^{\prime \prime} \mathrm{E} & \lambda \max =01^{\circ} 05^{\prime} 22 " \mathrm{E} \\
\phi \min =33^{\circ} 34^{\prime} 48^{\prime \prime} \mathrm{N} & \phi \max =33^{\circ} 46^{\prime} 07^{\prime \prime N}\end{array}$ \\
\hline
\end{tabular}


Tableau 1 : Exemple de Métadonnées d'une image ALSAT2

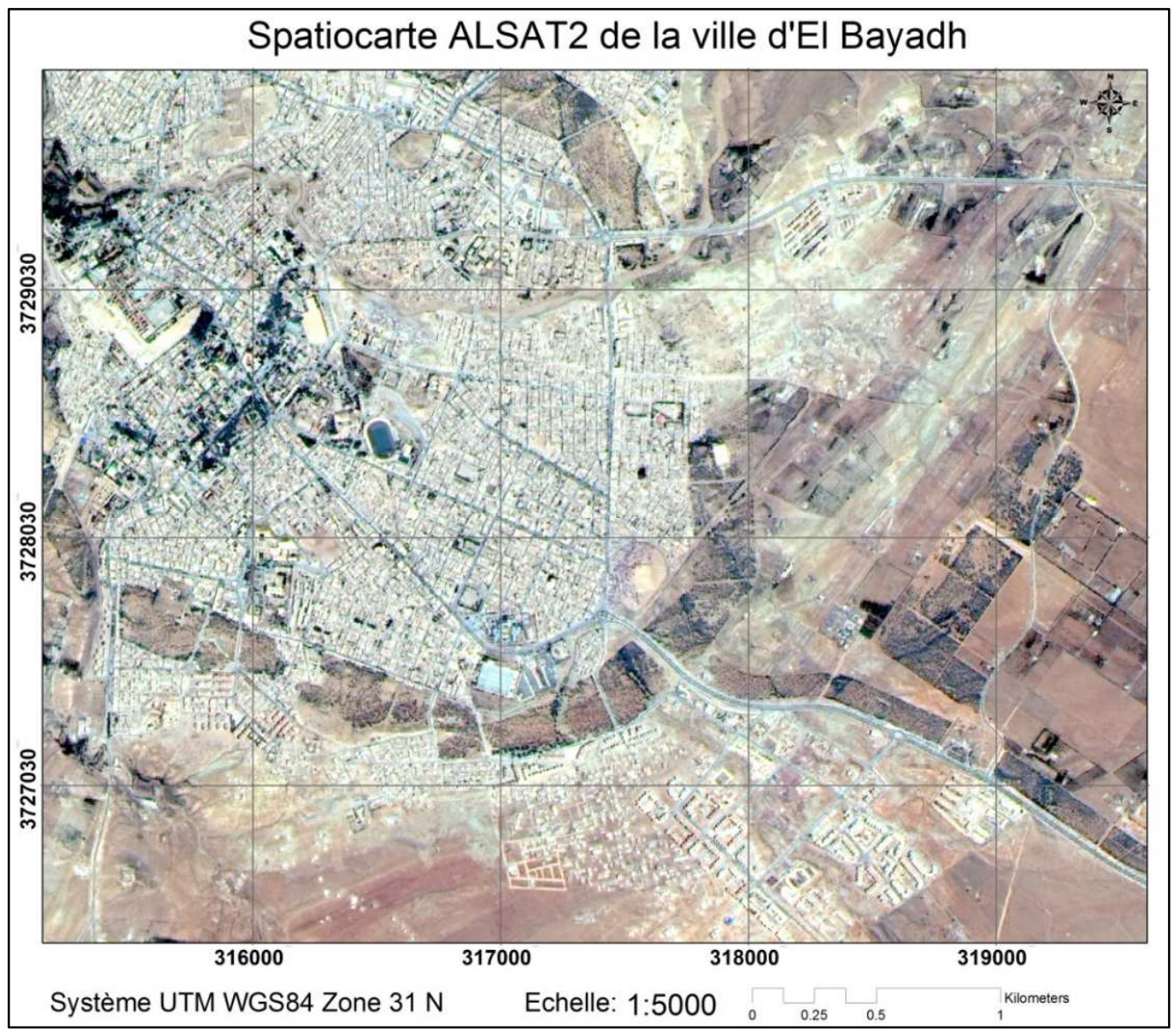

Figure 5 : Spatiocarte à partir d'une image ALSAT2 multispectrale de la ville d'El Bayadh.

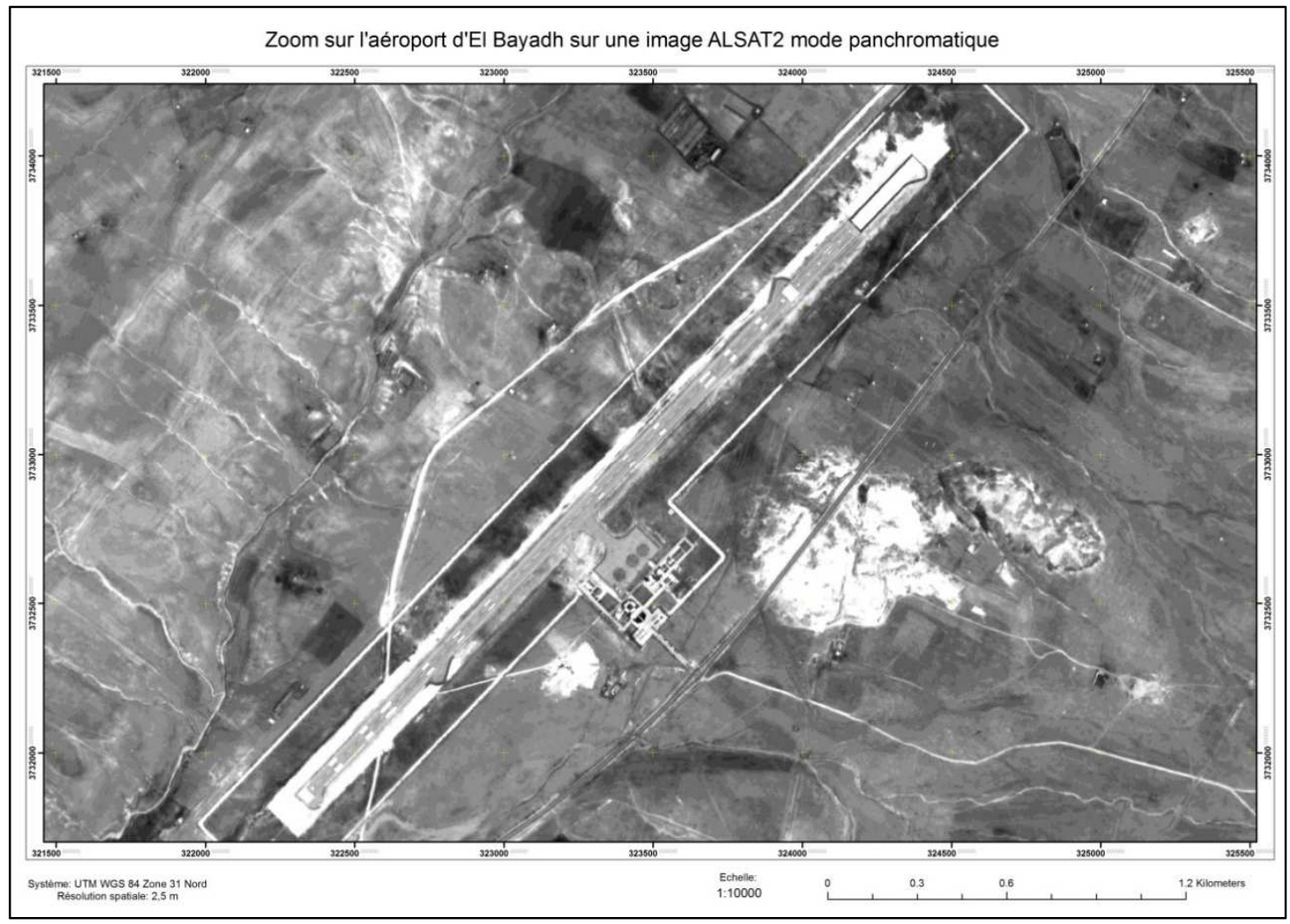


Figure 6 : Spatiocarte d'une image ALSAT2 panchromatique de l'aéroport d'El Bayadh (Système UTM WGS 84 Zone 31 Nord, $\left.x_{\min }=321000, y_{\min }=3732000, x_{\max }=325500, y_{\max }=3734000 \mathrm{~m}\right)$.

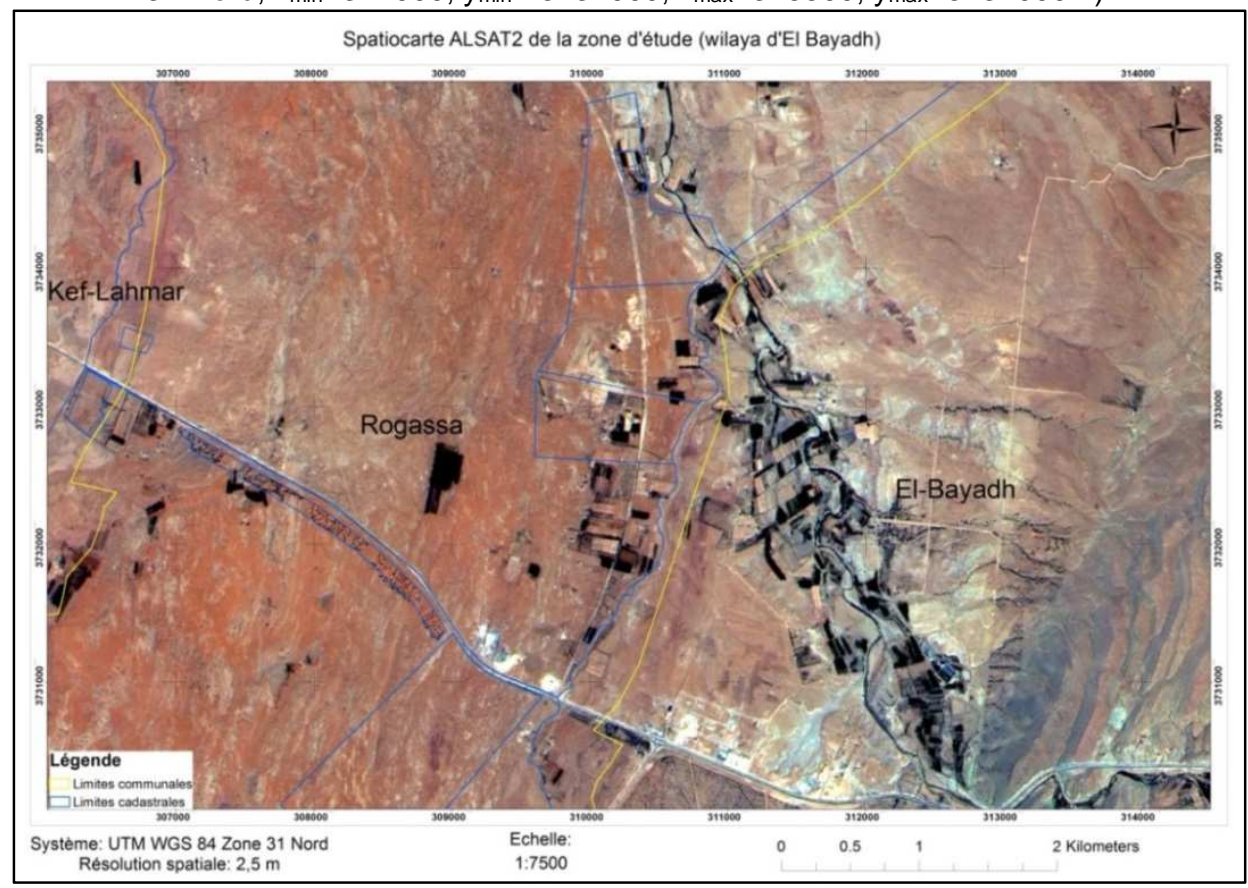

Figure 7 : Spatiocarte de la zone d'enquête cadastrale ( $\left.x_{\min }=307000, y_{\min }=3731000, x_{\max }=314000, y_{\max }=3735000 \mathrm{~m}\right)$.

Pour démontrer l'efficacité des images ALSAT2 en matière d'extraction de l'information (utile pour la mise à jour du cadastre steppique) un jeu de données des bases graphiques Version 1.6 (dernière mise à jour en 2013) a été récupéré auprès de l'Agence Nationale du Cadastre qui couvre la commune d'El Bayadh (chef-lieu de la wilaya) et la commune de Rogassa.

Références cadastrales des zones choisies

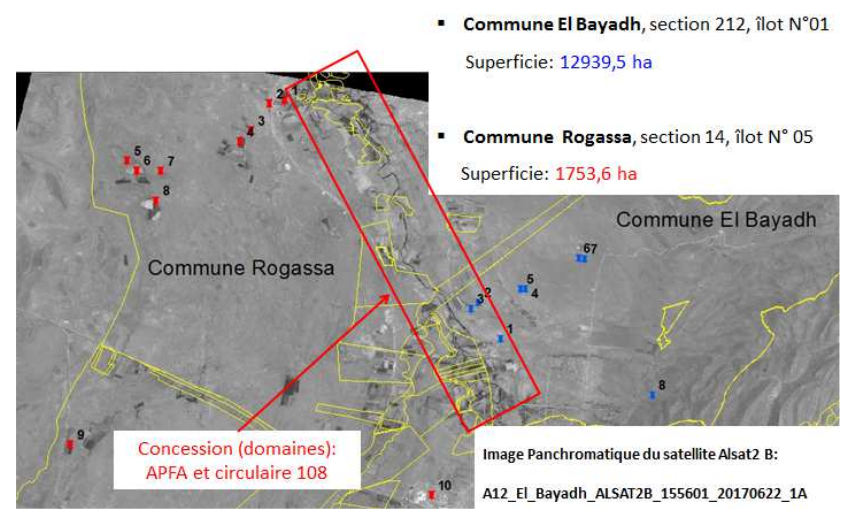

Figure 8 : Références cadastrales des sites choisis $\left(\mathrm{x}_{\text {centroide }}=311398, \mathrm{y}_{\text {centroide }}=3732773 \mathrm{~m}\right)$.

\section{Méthodologie et résultats}

La disponibilité de données haute résolution à partir d'une plate-forme satellitaire a ouvert de nouvelles possibilités pour la cartographie et la mise à jour du cadastre (P. Jayaprasad et al, 2006). Le paysage steppique est marqué par la présence de détails inférieurs au seuil théorique d'extraction de plusieurs objets, par exemple l'objet bâti isolé qui est un élément pertinent d'appréciation de l'omission de la présence des propriétés recherchées (propriétés

privées, de l'Etat occupées dans le cadre de concessions ou Arch). A cet effet, nous avons opté pour des méthodes qui intègrent, en plus des dimensions spectrales, les informations texturales et géométriques, parmi lesquelles figure l'approche orientée objet (A. Puissant, 2003). La méthodologie suivie repose sur le principe de tester plusieurs classifieurs qui donnent généralement des informations certaines (redondantes) et d'autres en confusion (complémentaires). Le but recherché est la formulation de règles de connaissances permettant l'identification et l'extraction des éléments pertinents à la maintenance du cadastre steppique dans le cadre d'une démarche de classification orientée objet.

Durant tous les traitements, le choix des échantillons était en adéquation avec la nomenclature Spot Théma définie à une résolution spatiale équivalente à l'image support d'étude. (Spot Théma est un produit d'occupation du sol à l'échelle des agglomérations, proposé sur la France métropolitaine et décliné en 8, 29 et 46 classes. II s'agit d'une base de données vectorielles produite à partir de l'interprétation d'images satellites SPOT 1 à 5 ou équivalent, d'une résolution de 2,5 et $20 \mathrm{~m}$, Astrium GEO Information Services, 2011).

Ainsi la notion de zone exclue (omise) du cadastre steppique a été redéfinie dans le cadre de ce travail de manière à prendre en considération toute possibilité d'existence de propriété privée dans le territoire cadastré. Initialement cette notion portait sur les agglomérations, les zones comportant des exploitations agricoles ou tous les établissements (quelle qu'en soit la 
nature) qui sont seulement «délimités» suivant une vectorisation de leurs contours en raison de la densité des unités foncières qui la composent. Elles figurent dans le plan cadastral en tant que périmètres non soumis aux opérations du cadastre saharien ou steppique. Elles seront cadastrées ultérieurement selon les modalités techniques habituelles basées sur les orthophotoplans et restitutions photogrammétriques.

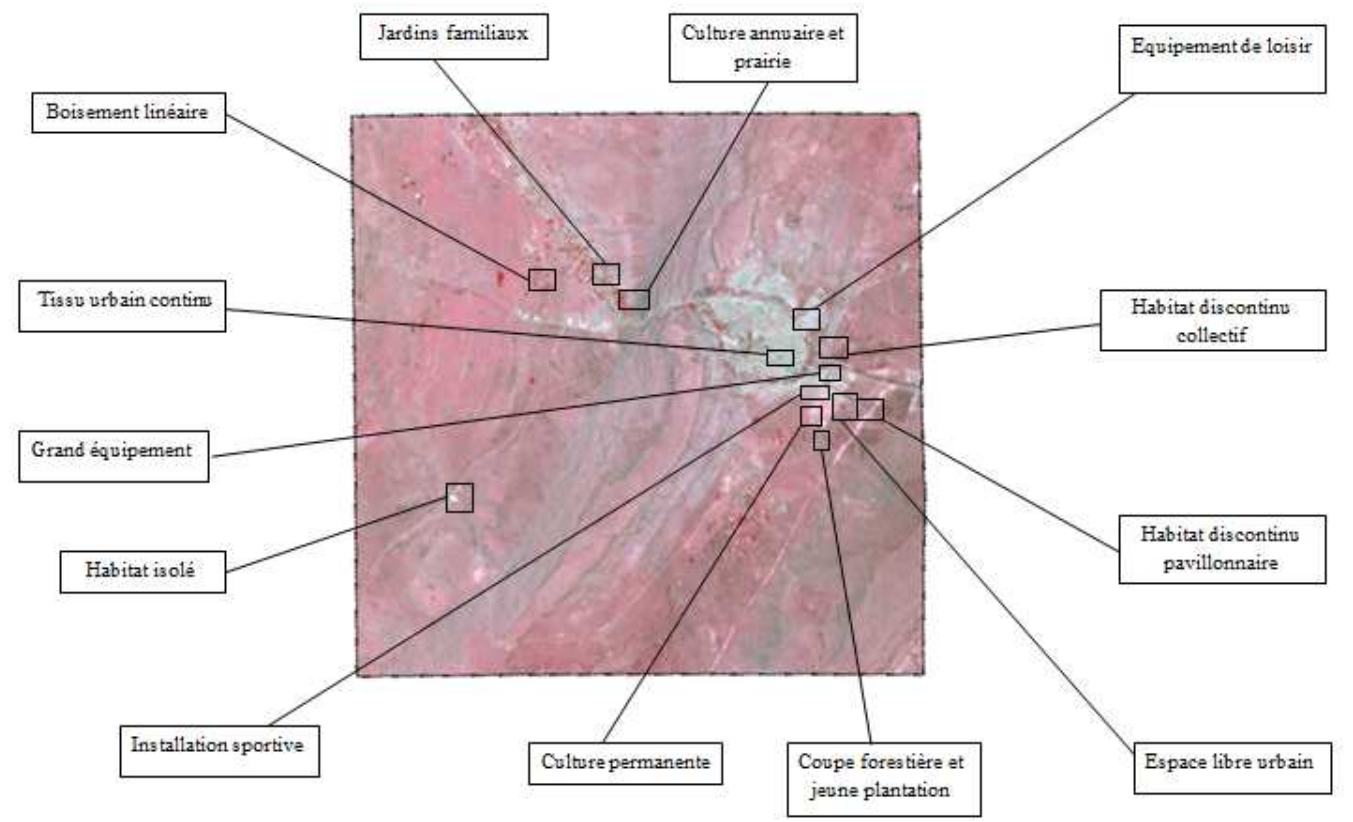

Figure 9 : Répartition spatiale sur l'image ALSAT2 des échantillons choisis selon la nomenclature d'objets Spot-Théma (Système UTM WGS 84 Zone 31 Nord, $x_{\min }=301500, y_{\min }=3720000, x_{\max }=323000, y_{\max }=3740000 \mathrm{~m}$ ).

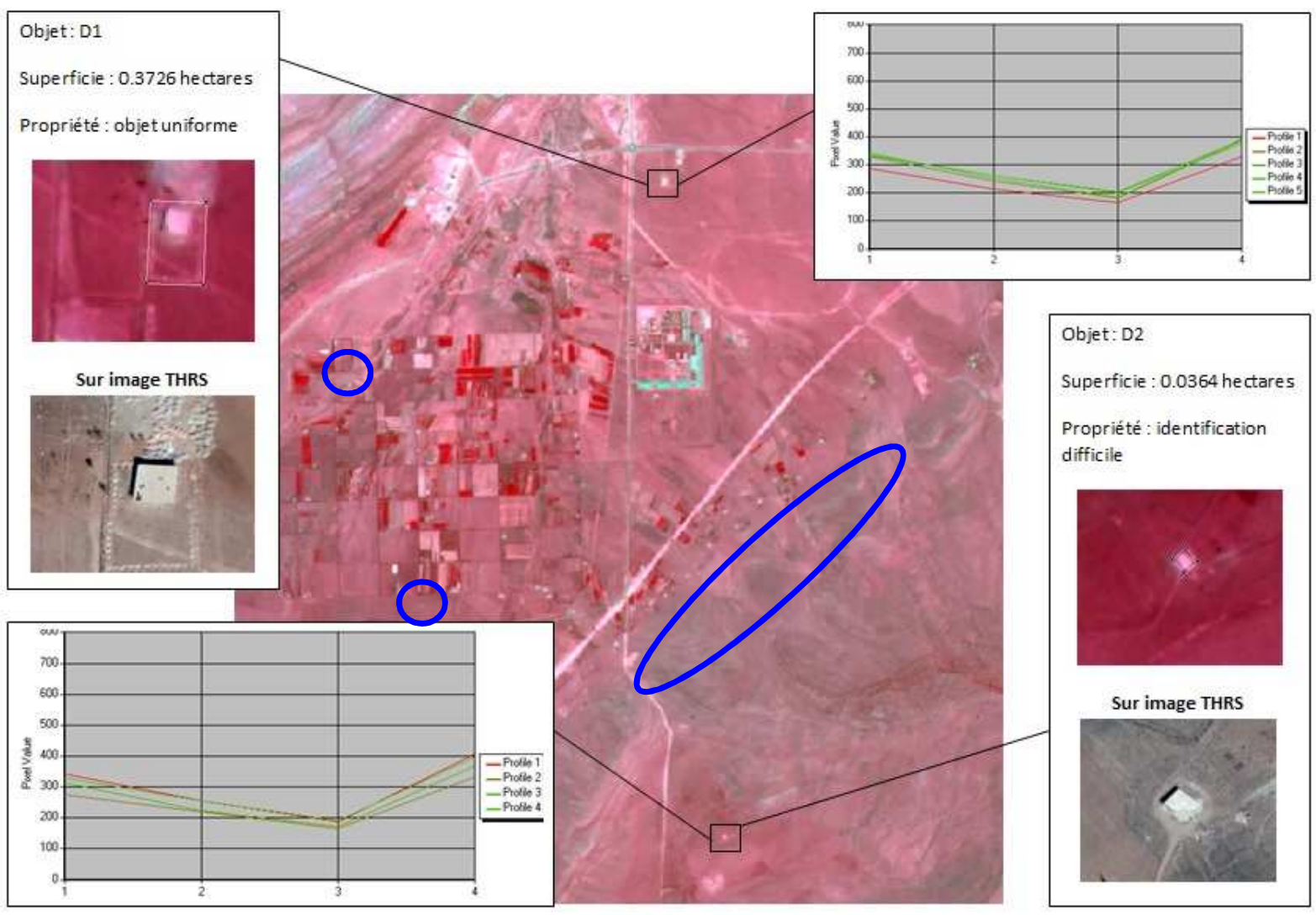

Figure 10 : Exemples de propriétés spectrales de quelques échantillons choisis selon la nomenclature d'objets SpotThéma (leur identification avec agrandissement à partir de l'image ALSAT2 et sur images THRS type Google-Earth). Les cercles bleus correspondent à des objets bâtis qui ne peuvent pas être considérés comme isolés en raison de leur proximité avec des routes ou leurs positions à l'intérieur de parcelles (facilement identifiables). (Système UTM WGS 84 Zone 31 Nord, point en haut $x=315650, y=3724500$, point en bas $x=316014, y=3720501 \mathrm{~m}$ ). 
La première lecture des images ALSAT2 donne l'impression qu'il s'agit d'un paysage à trois grandes classes thématiques: urbain, sol et végétation. Alors qu'en réalité c'est un milieu (espace) marqué par la dominance du bâti isolé et des cultures qu'on peut faire correspondre à des jardins familiaux de la nomenclature Spot Théma (figure 9).

Notre objectif ici n'est pas de tester les méthodes de classification pour une production cartographique ordinaire, qui doit obéir aux normes de représentation et de définition des objets sur des plans, mais plutôt d'extraire l'information sur ces petits objets 'bâtis isolés (niveau d'identification suffisant) ou parcelles (cartographie à une échelle 1/10000 à 1/25000)' qui fourniront des informations sur la présence des propriétés privées ou des biens étatiques occupés par des personnes physiques. Par souci d'atteindre une certaine qualité, nous avons veillé à la bonne définition des échantillons d'extraction. Ainsi, nous avons testé les méthodes standards dans le domaine des classifications d'image pour bien cerner la variation spectrale des différents objets. Ensuite, nous les avons intégrées dans un processus de fusion orientée objet pour avoir des résultats avec un taux minimum de confusion thématique.

L'organigramme présenté dans la figure 11 résume toute la chaine de traitement allant de l'image support d'étude en passant par la nomenclature des objets et la définition des zones exclues (omises) jusqu'à l'obtention des résultats et leur validation.

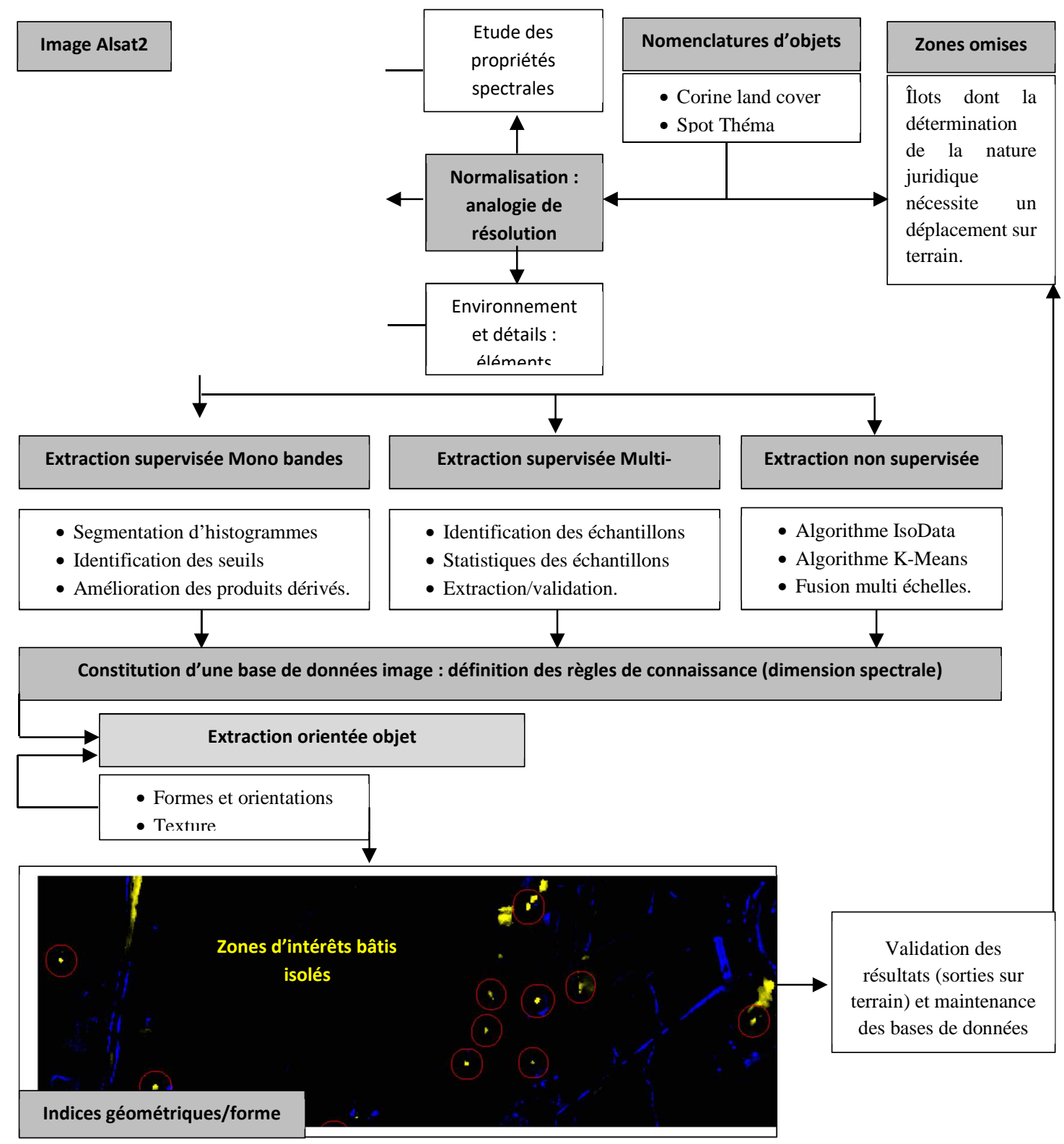

Figure 11 : Approche méthodologique développée pour l'identification des zones d'intérêt (îlots de propriété bâtis ou non bâtis) dans le cadre de la maintenance du cadastre steppique 


\subsection{Précision géométrique de l'imagerie ALSAT2}

Plusieurs normes relatives à la notion de précision de la position existent dans la littérature (ex : P. A. Handbook, 1999) La norme NMAS (United States National Map Accuracy Standards) appliquées aux USA (F.G.D.Committee, 1998), qui est adaptée aux grandes étendues (cas de nos régions steppiques et sahariennes en Algérie). Elle a une relation entre l'indice CE90 et l'échelle de restitution. Elle est donnée pour des échelles plus grandes que 1/20 000 par:

\section{$E=C E 90 \times 100 /(2,54 \times 0,03333)$}

Elle utilise l'indice CE90 dont l'erreur a un intervalle de confiance de $90 \%$ pour évaluer la qualité des documents. Cinq points GPS ont servi à la détermination de l'échelle de l'image ALSAT2. Ces points sont exclus du calcul de l'orientation externe.

\begin{tabular}{|c|c|c|c|}
\hline \multicolumn{2}{|c|}{ Coordonnées GPS } & \multicolumn{2}{c|}{$\begin{array}{c}\text { Coordonnées Ortho-image } \\
\text { ALSAT2 }\end{array}$} \\
\hline$X$ & $Y$ & $X$ & $Y$ \\
\hline 318892.67 & 3733998.59 & 318890.00 & 3734001.89 \\
\hline 319194.83 & 3722897.33 & 319197.43 & 3722894.64 \\
\hline 309539.50 & 3719456.88 & 309537.75 & 3719456.87 \\
\hline 315693.63 & 3716341.13 & 315693.79 & 3716343.18 \\
\hline 312979.54 & 3709923.89 & 312980.97 & 3709921.24 \\
\hline
\end{tabular}

Tableau 2 : Coordonnées des points GPS et Orthoimage ALSAT2 utilisées pour la détermination de l'échelle.

\begin{tabular}{|c|c|c|c|c|c|}
\hline Ecart X & Ecart Y & Ecart D & $\sigma x$ & бy & $\sigma l$ \\
\hline 2,675 & $-3,298$ & 4,24 & 2,18 & 2,71 & 3,48 \\
\hline$-2,597$ & 2,697 & 3,74 & \multirow{4}{*}{\multicolumn{3}{|c|}{ CE90 $=7,46$ et $E=8816$}} \\
\hline 1,755 & 0,014 & 1,75 & & & \\
\hline$-0,153$ & $-2,048$ & 2,05 & & & \\
\hline$-1,422$ & 2,651 & 3,00 & & & \\
\hline
\end{tabular}

Tableau 3 : Détermination de l'échelle cartographique de l'image ALSAT2 support d'étude.

L'échelle déterminée par application de la norme NMAS est $1 / E=1 / 9000$ et qui ne concerne que la précision de position sans relation avec l'identification des objets qui dépend de la résolution spatiale de l'image.

\subsection{Capacité informative de l'imagerie ALSAT2}

Une interprétation spectrale a été faite en examinant plusieurs échantillons présentés :
- Au niveau des statistiques par pixel : sont illustrées par le tracé des signatures spectrales dans les quatre bandes (bleu, vert, rouge et proche infrarouge) et ne représentent qu'une variation des pixels isolés (figure10) sans tenir compte des variations du voisinage et de la totalité des pixels qui forme l'objet. Les signatures confirment l'appartenance des pixels à la même classe thématique mais n'indiquent pas la confusion spectrale entres les pixels testés.

- Au niveau des statistiques par objet : contrairement au cas précédent les statistiques zonales ou par objet peuvent révéler l'hétérogénéité spectrale de l'objet et son voisinage. Cela dépend d'une part, du nombre de pixels qui le composent, et d'autre part, de la nature de l'objet lui-même.

\subsubsection{Extraction supervisée Mono bande}

Dans le but de traiter les bandes spectrales individuellement en fonction des propriétés intrinsèques de chaque objet, la technique d'extraction est personnalisée. Le travail se fait manuellement et nécessite des connaissances préalables sur le comportement spectral de chaque objet dans chaque bande. Le calcul d'indice tel que le NDVI permet une séparation thématique qui peut servir à une réduction de la probabilité de confusion interclasses.

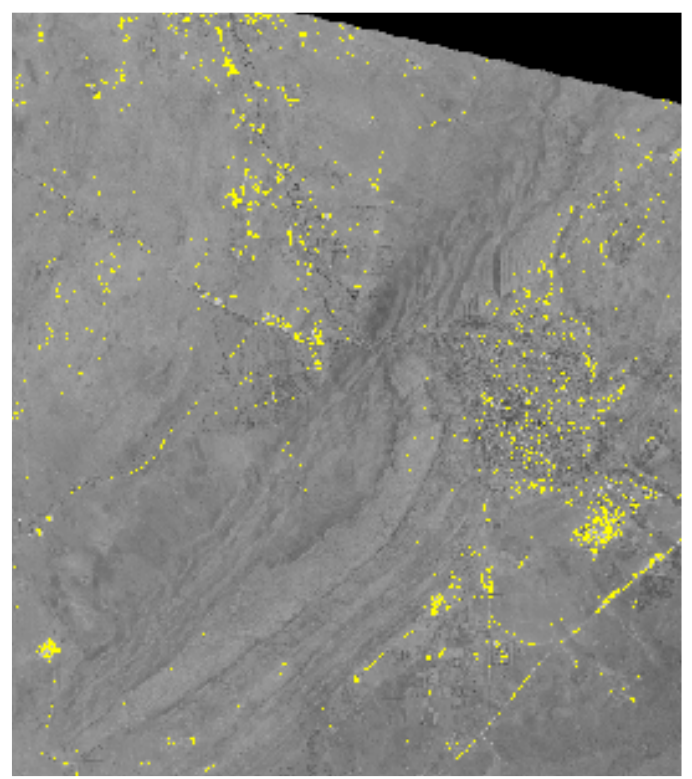

Figure 12 : Exemple de confusions thématiques (pixels en jaune) dues à l'extraction mono-bande ( Xentroide $\left.=312623, \mathrm{y}_{\text {centroide }}=3729383 \mathrm{~m}\right)$.

\subsubsection{Extraction Multi-bandes}

Extraction non supervisée

Dans cette phase de traitements deux algorithmes standards sont utilisés (celui d'Isodata et du K-Means) afin de mettre en évidence les variations spectrales des deux objets avec un nombre de classes : 5 , sur 10 et 15 . 
La comparaison des deux algorithmes de classification non supervisée utilisés nous a permis de constater :

- Une extraction moins confuse pour l'objet végétation,

- Une extraction de l'objet bâti avec un taux de confusion plus ou moins important et à des degrés différents,

- Pour les deux algorithmes l'augmentation du nombre de classes améliore légèrement la qualité mais en même temps change les seuils des confusions,

La qualité d'extraction de l'objet bâti par algorithme d'Isodata est relativement meilleure que celle obtenue par l'algorithme K-Means.

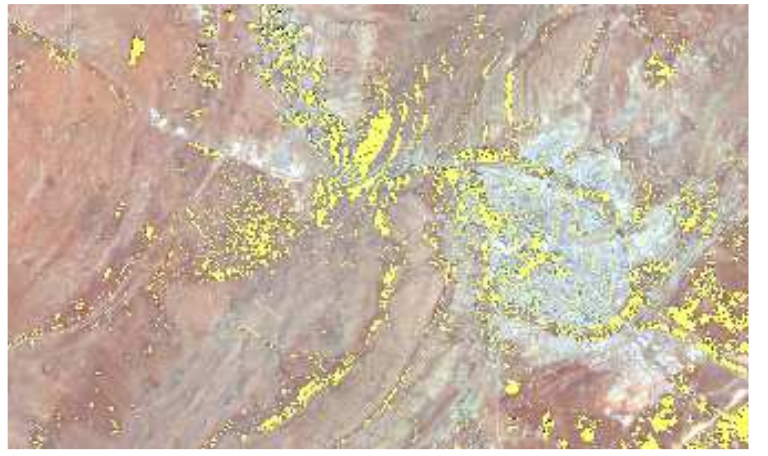

Figure 13 : Exemple de confusions thématiques (pixels en jaune) dues à l'extraction par application de l'algorithme K-Means ( $x_{\text {centroide }}=312819 \mathrm{~m}$, $\left.\mathrm{y}_{\text {centroide }}=3729944 \mathrm{~m}\right)$.

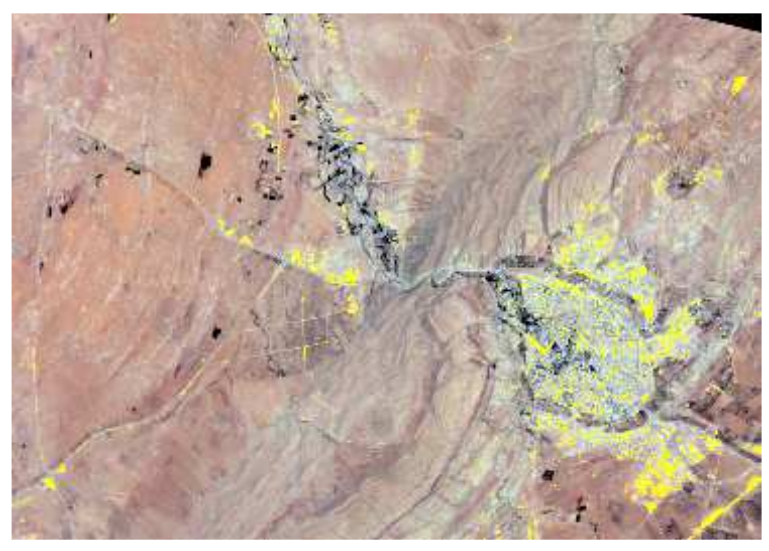

Figure 14 : Exemple de confusions thématiques (pixels en jaune) dues à l'extraction par application de l'algorithme ISODATA ( $x_{\text {centroide }}=312819 \mathrm{~m}$, $\left.\mathrm{y}_{\text {centroide }}=3729944 \mathrm{~m}\right)$.

\section{Extraction par classification supervisée}

Pour choisir les échantillons de classification deux contrôles sont nécessaires :

- Son identité spectrale: par le tracé de la signature qui confirme son appartenance,

- Son histogramme: qui montre s'il est en confusion avec d'autres objets ou non.

L'algorithme choisi pour lancer la classification est celui du maximum de vraisemblance. Contrairement au cas de la végétation, l'objet bâti présente un taux de confusion important. Les opérations de nettoyage se limitent à des simples requêtes sur les superficies.

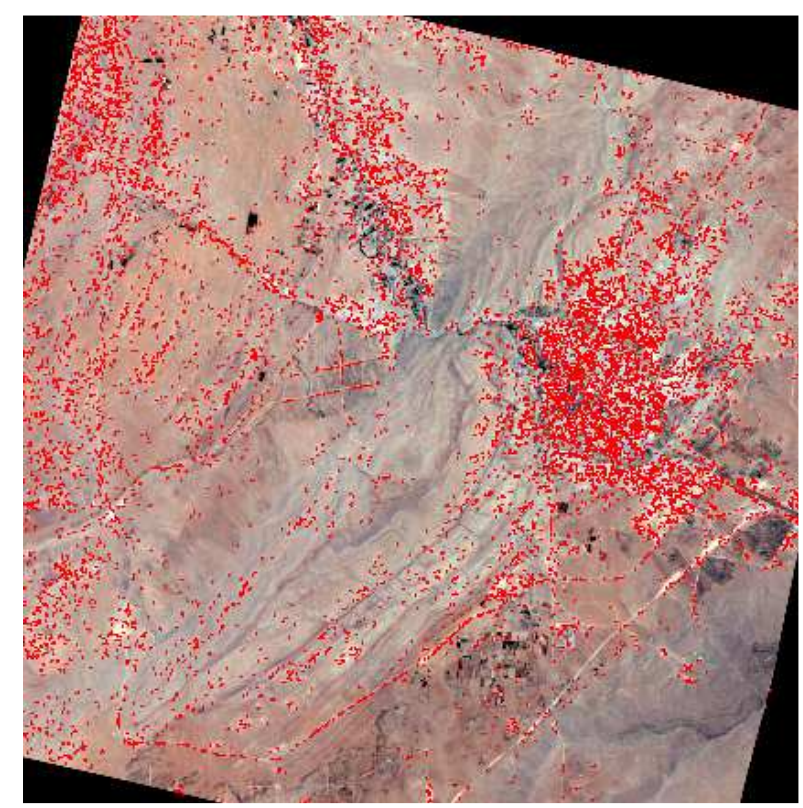

Figure 15 : Exemple de confusions thématiques (pixels en rouge) dues à l'extraction par application de la classification supervisée par maximum de vraisemblance. $\left(x_{\min }=301600, y_{\min }=3719520\right.$, $\left.x_{\max }=322926, y_{\max }=3735924 \mathrm{~m}\right)$.

\section{Synthèse}

L'analyse des techniques d'extraction testées montre que pour chaque résultat il y' a une information certaine sur les deux objets (végétation et bâti). En revanche l'incertitude est fortement liée aux dimensions de l'objet au sol et à sa nature (voir annexe).

L'analyse de l'extraction brute de l'objet bâti (illustrée par le graphe de la figure 16) confirme l'hypothèse que le résultat représente un maximum d'objets avec une forte probabilité de confusion.

Cette hypothèse n'est pas toujours fiable pour le cas de la végétation. Néanmoins, l'hétérogénéité des espèces rend souvent la confusion significative. 


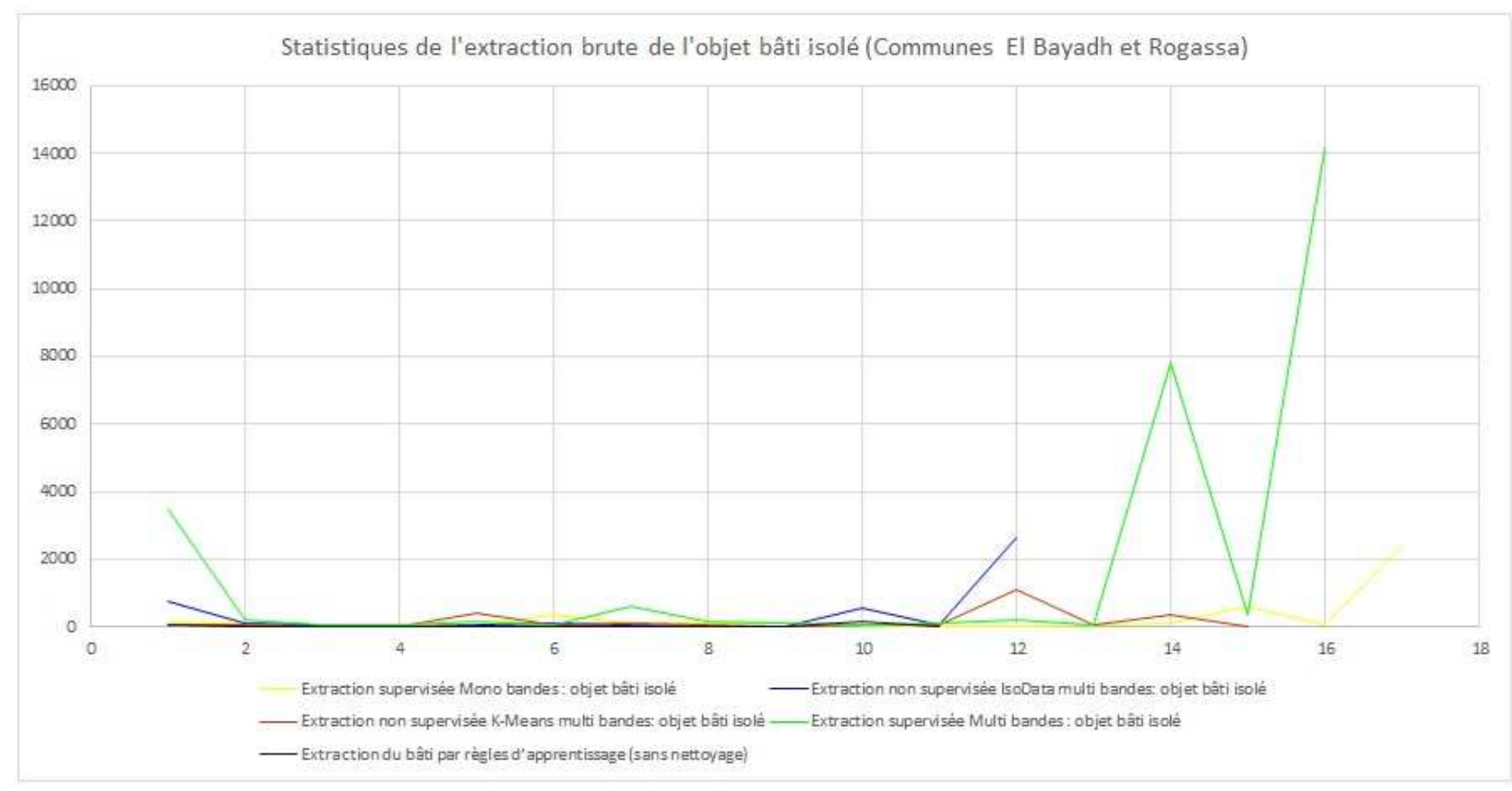

Figure 16 : Statistiques des nombres d'objets bâtis extraits par différents algorithmes de classification

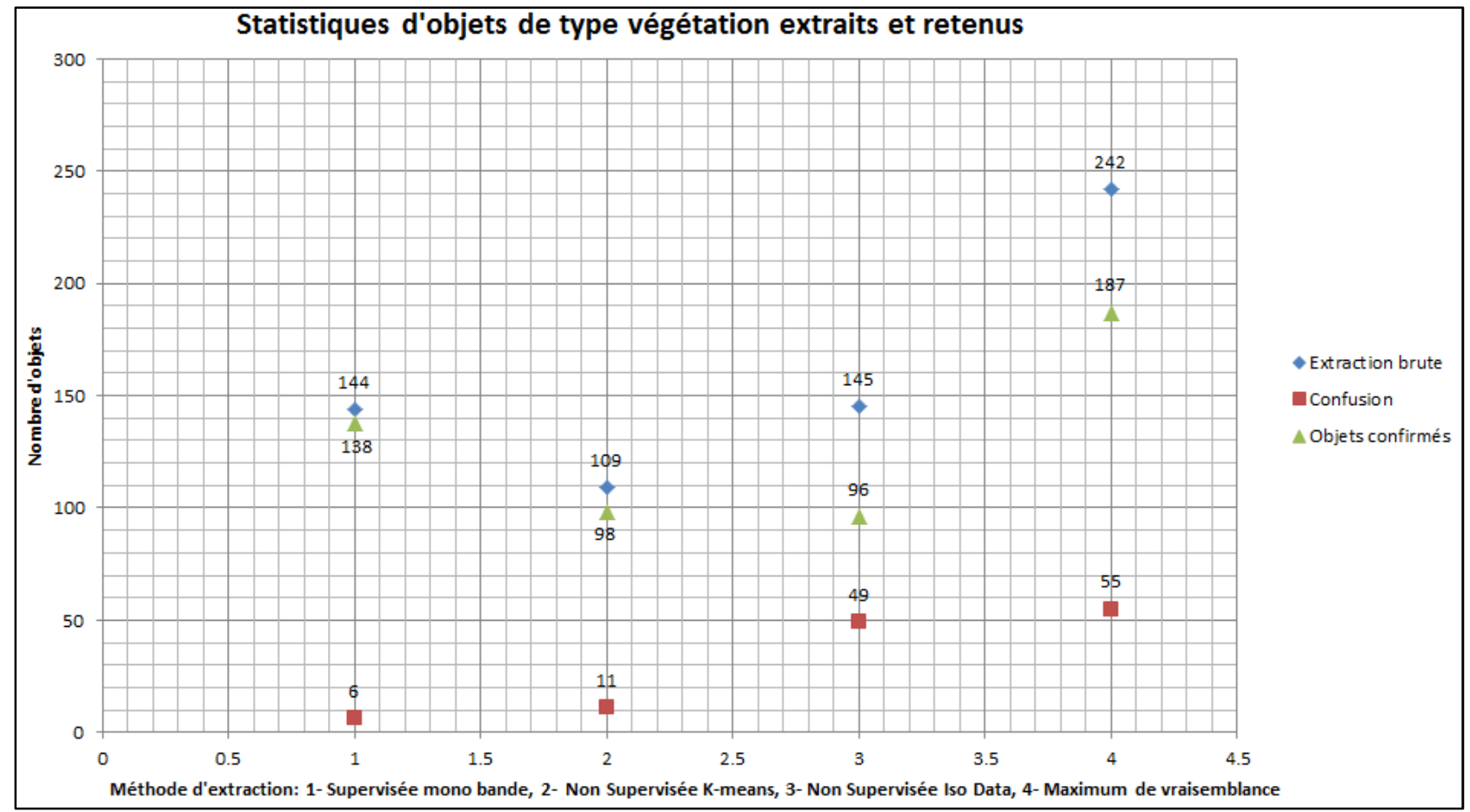

Figure 17 : Comparaison des statistiques d'extraction de l'objet végétation

\subsubsection{Extraction par approche orientée objet}

Les objets avec les mêmes revêtements nous fourniront une même signature spectrale sur une image satellitaire mais seront reconnaissables par leurs formes et textures respectives. Ce problème est lié à la variabilité radiométrique, spatiale et spectrale.

Ainsi, la finesse de la résolution spatiale des images haute résolution telle que ALSAT2, exige de travailler dans un espace à plusieurs dimensions et non pas uniquement limité à la seule dimension spectrale.

Dans cette partie, nous avons opté pour une solution permettant l'intégration d'un certain nombre de règles de connaissances afin de guider l'algorithme à une identification précise de l'objet à extraire. Ces connaissances sont définies pour chaque segment à des échelles différentes. 


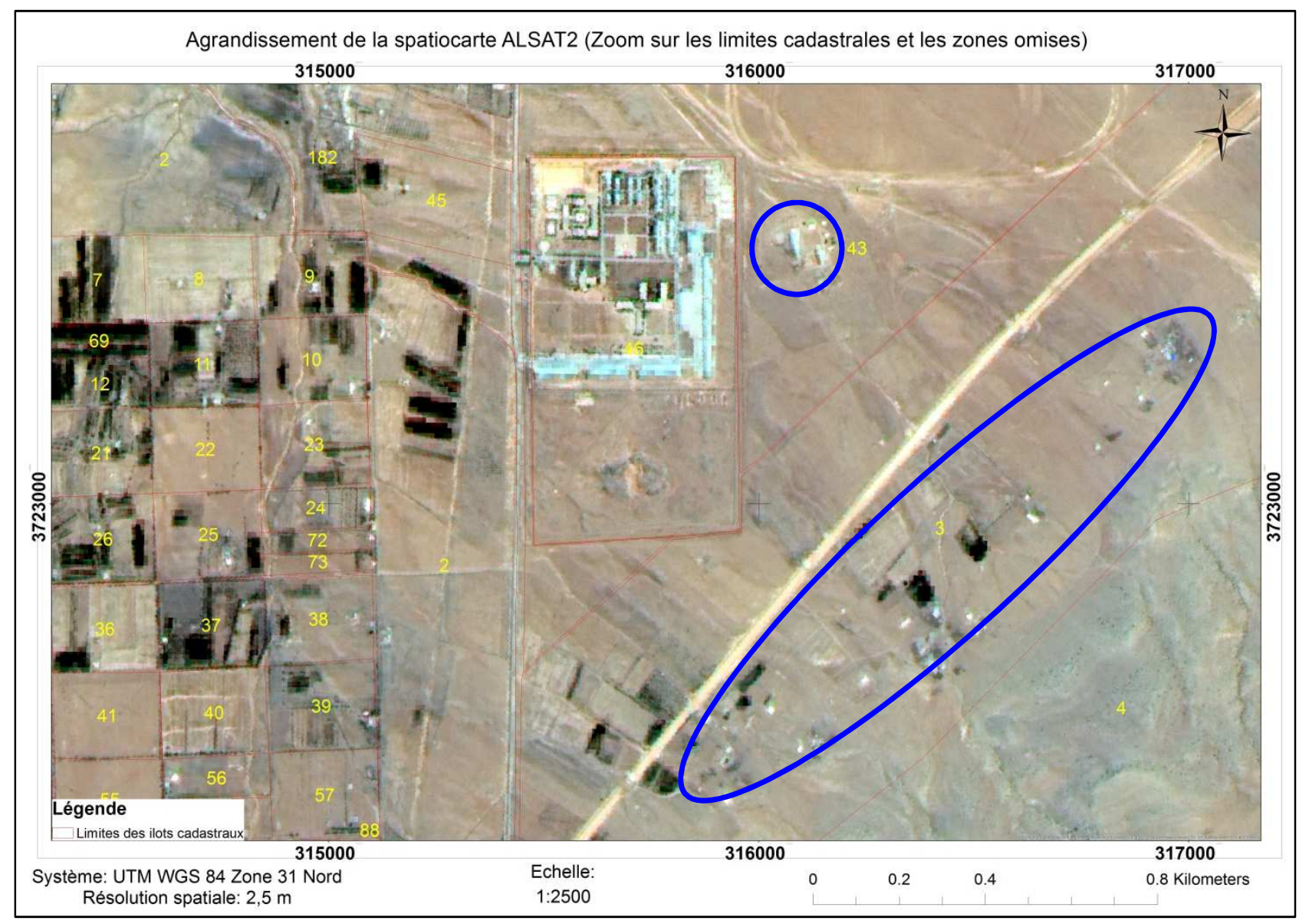

Figure 18 : Agrandissement de la Spatiocarte ALSAT2 au 1/2500 sur laquelle sont encerclés en bleu des objets bâtis et des parcelles omis qui appartiennent à l'îlot 3 et 43 cadastrés au nom de l'état.

Les imagettes illustrées ci-dessous sont extraites à partir de la Spatiocarte pour mettre en exergue les objets extraits (végétaux en couleur verte et bâti en magenta).

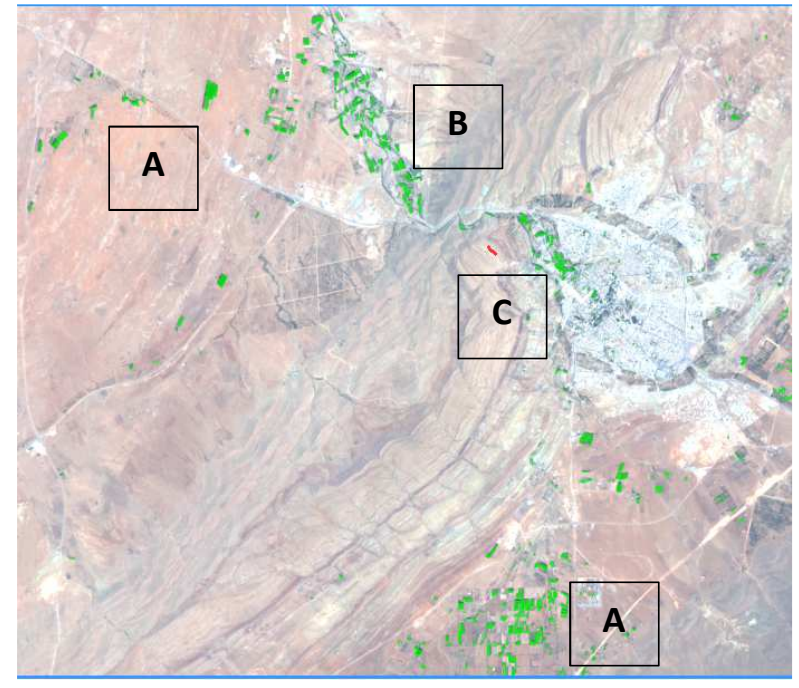

Figure 19 : Exemples de parcelles extraites $A$ (parcelles dispersées), B (Exploitations agricoles collectives $\mathrm{EAC}), \mathrm{C}$ (végétation périurbaine). $\left(\mathrm{X}_{\text {centroide }}=311700 \mathrm{~m}, \mathrm{y}_{\text {centroide }}=3728632 \mathrm{~m}\right)$.

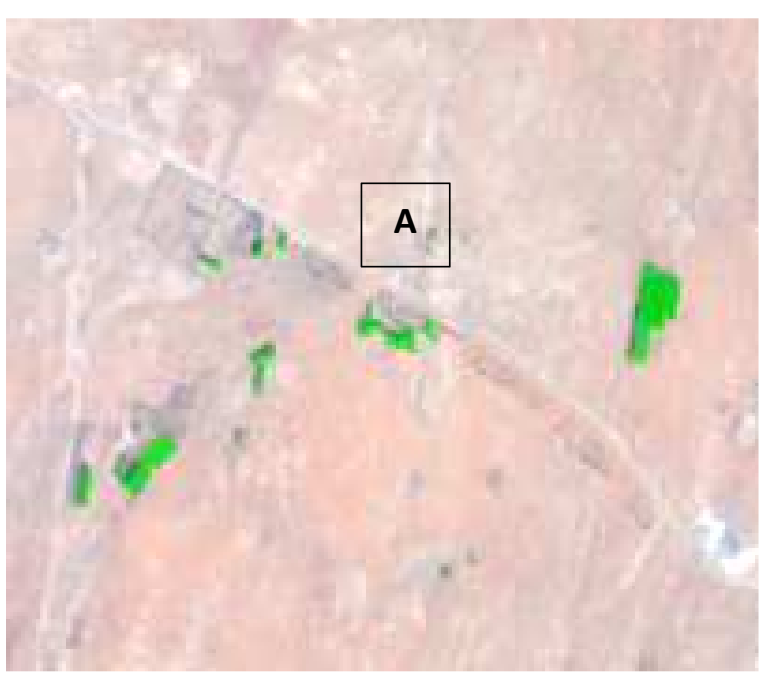

Figure 20 : Zoom sur la classe A (en couleur verte) niveau d'extraction équivalent à l'échelle $1 / 10000$ ( $X_{\text {centroide }}=307120 \mathrm{~m}, y_{\text {centroide }}=3732058 \mathrm{~m}$ ). 


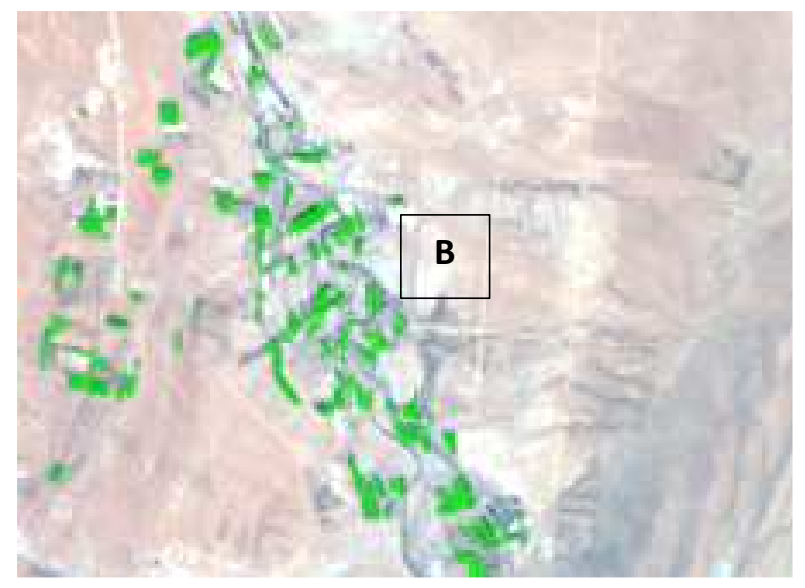

Figure 21 : Zoom sur la classe B (en couleur verte) niveau d'extraction équivalent à l'échelle $1 / 10000$ $\left(x_{\text {centroide }}=312164 \mathrm{~m}, \mathrm{y}_{\text {centroide }}=3732177 \mathrm{~m}\right)$.

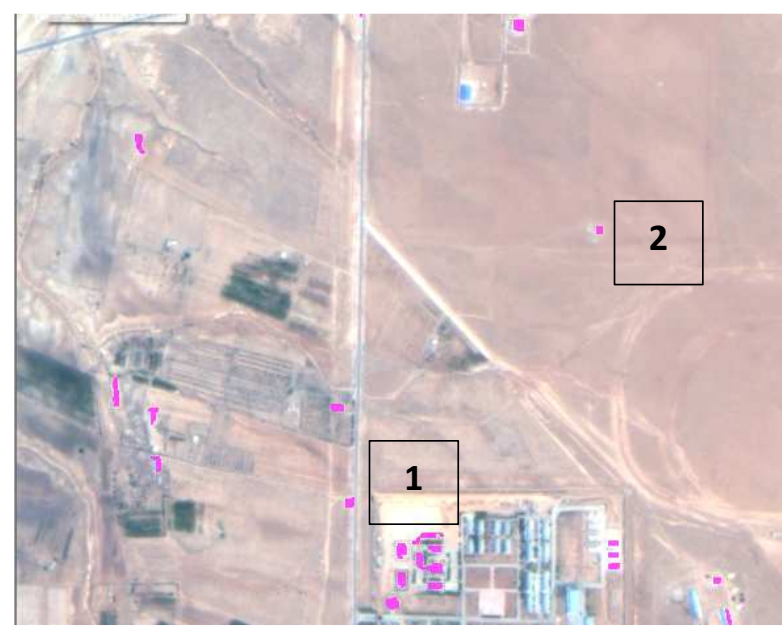

Figure 22 : Exemples du bâti identifié (sous forme de groupement de type industriel ou autre, c'est la classe 01 , ou isolé classe 02$)\left(x_{\text {centroide }}=315500 \mathrm{~m}\right.$, $\left.\mathrm{y}_{\text {centroide }}=3724045 \mathrm{~m}\right)$.

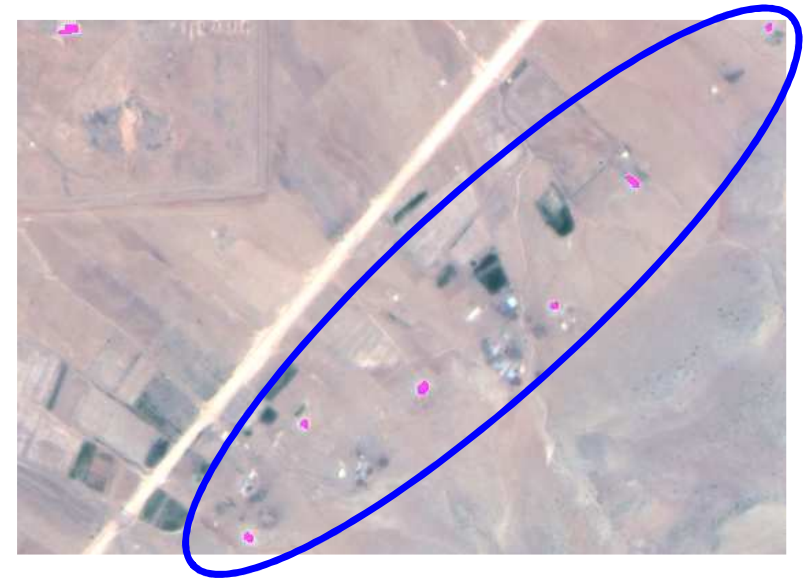

Figure 23: Exemples de bâtis isolés extraits classe 2 sur la figure 22 (objets encadrés en bleu sur la figure 10). $\left(X_{\text {centroide }}=316200 \mathrm{~m}\right.$, ycentroide $\left.=3722751 \mathrm{~m}\right)$.
L'extraction en couleur magenta est qualitative. Le niveau de zoom de l'image est à l'échelle 1/7500 mais les objets extraits ne sont pas représentés dans les vraies dimensions. En agrandissant on perd la qualité visuelle de l'image. Pour les échelles plus grandes que $1 / 5000$ la visualisation devient floue (figures 24). Mais nous pouvons toujours identifier des segments de bâtis ou de routes (figure 25).

Ces résultats corroborent des résultats similaires obtenus dans d'autres études réalisées sur la base de l'imagerie SPOT5. La précision de l'identification dépend directement de la taille, de la forme du bien, de la topographie de la zone, du type de clôtures et de la couverture végétale présente sur la zone d'étude ainsi que de l'échelle de l'ortho-image utilisée dans le processus d'identification.

L'orthoimage SPOT 5 est considérée alors comme une alternative intéressante pour la méthodologie indirecte avec une faible variation topographique. Cependant, il est recommandé d'utiliser des méthodes d'arpentage traditionnelles pour les petites propriétés, le cadastre urbain et l'identification des bâtiments (M. Corlazzoli, 2004).

Pour une échelle de restitution de l'ordre du $10000^{\text {ème }}$, la résolution spatiale de l'image SPOT5 convient sauf pour la détection de certaines entités de petite taille et de texture particulière. (F. Gourmelon, 2005).

\subsubsection{Validation et discussion des résultats}

En plus des statistiques obtenues par application des différentes méthodes d'extraction (tableaux en annexe) et en dehors des avantages et des limites de chacune des méthodes, la validation des résultats est effectuée par leur confrontation à la réalité lors des sorties sur terrain. Les endroits visités correspondent aux références cadastrales suivantes :

- $\quad$ Commune Rogassa, Section 14 (îlots 05, 06 et 07): propriétés identifiées, visitées et enquêtées.

- $\quad$ Commune El Bayadh, Section 01 ( îlot $n^{\circ} 01$ et 08): propriétés identifiées, visitées et enquêtées.

La figure 26 montre des exemples sur la présence des propriétés privées et des propriétés Arch (appartenant à la même tribu) situées dans la commune de Rograssa. De même, la figure 27, montre des exemples sur la présence des propriétés Arch situant dans la commune El Bayadh. 


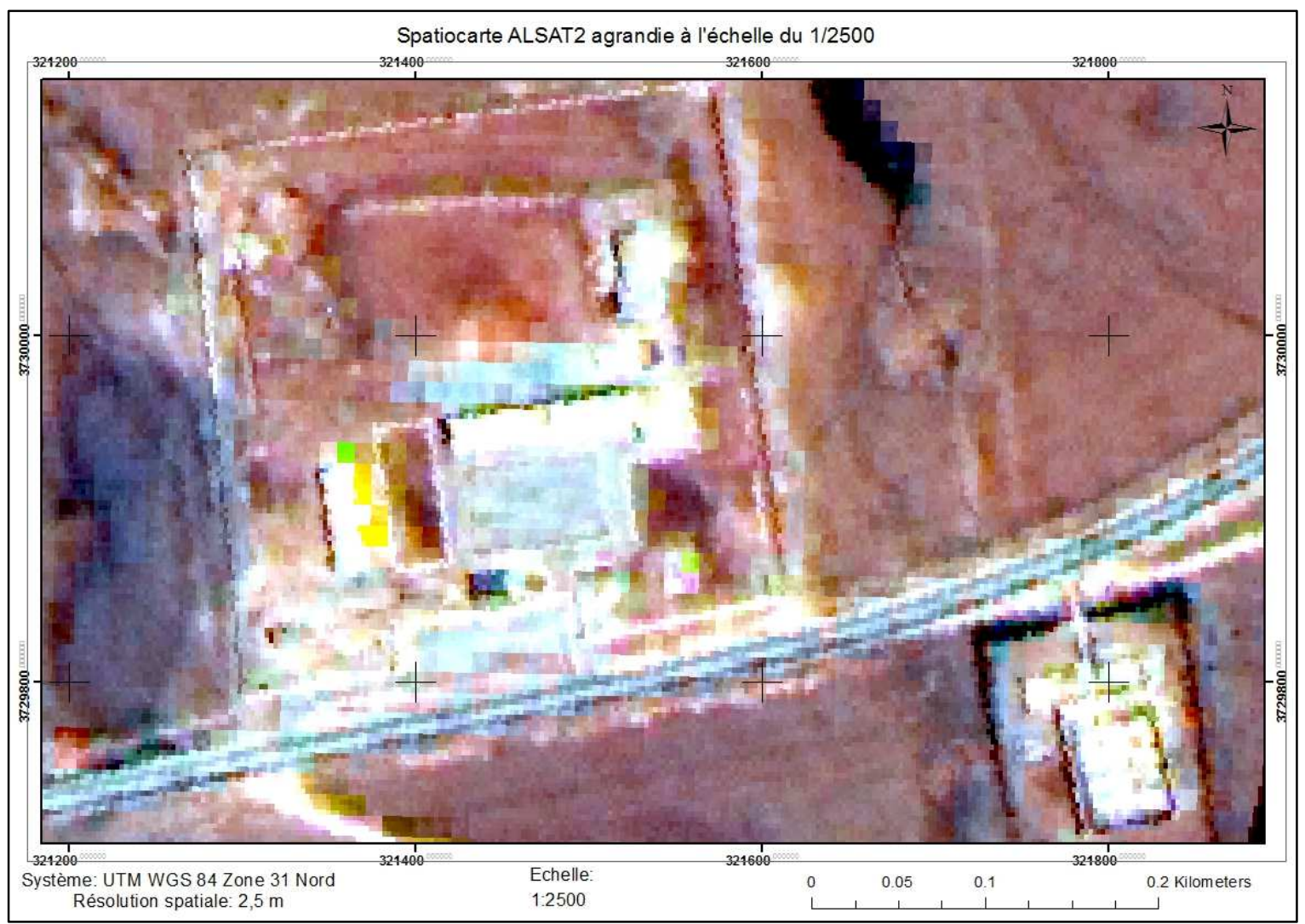

Figure 24 : Dégradation de la qualité visuelle de l'ortho-image à l'échelle 1/2500 (niveau de zoom différent de celui de la figure 18).

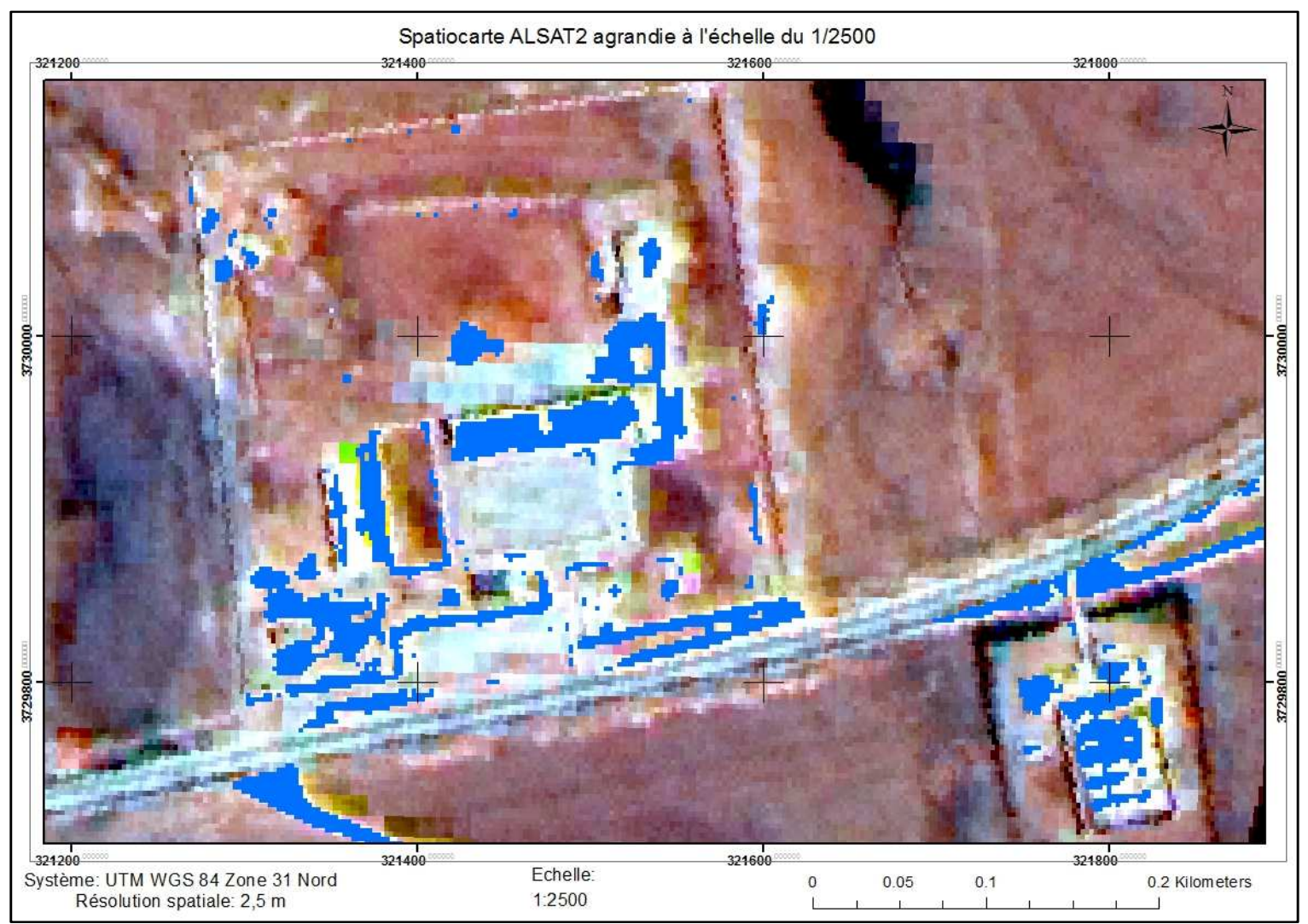

Figure 25 : Identification des segments de bâtis et de routes (en couleur bleu) à partir de l'image ALSAT2 affichée à l'échelle 1/2500. 


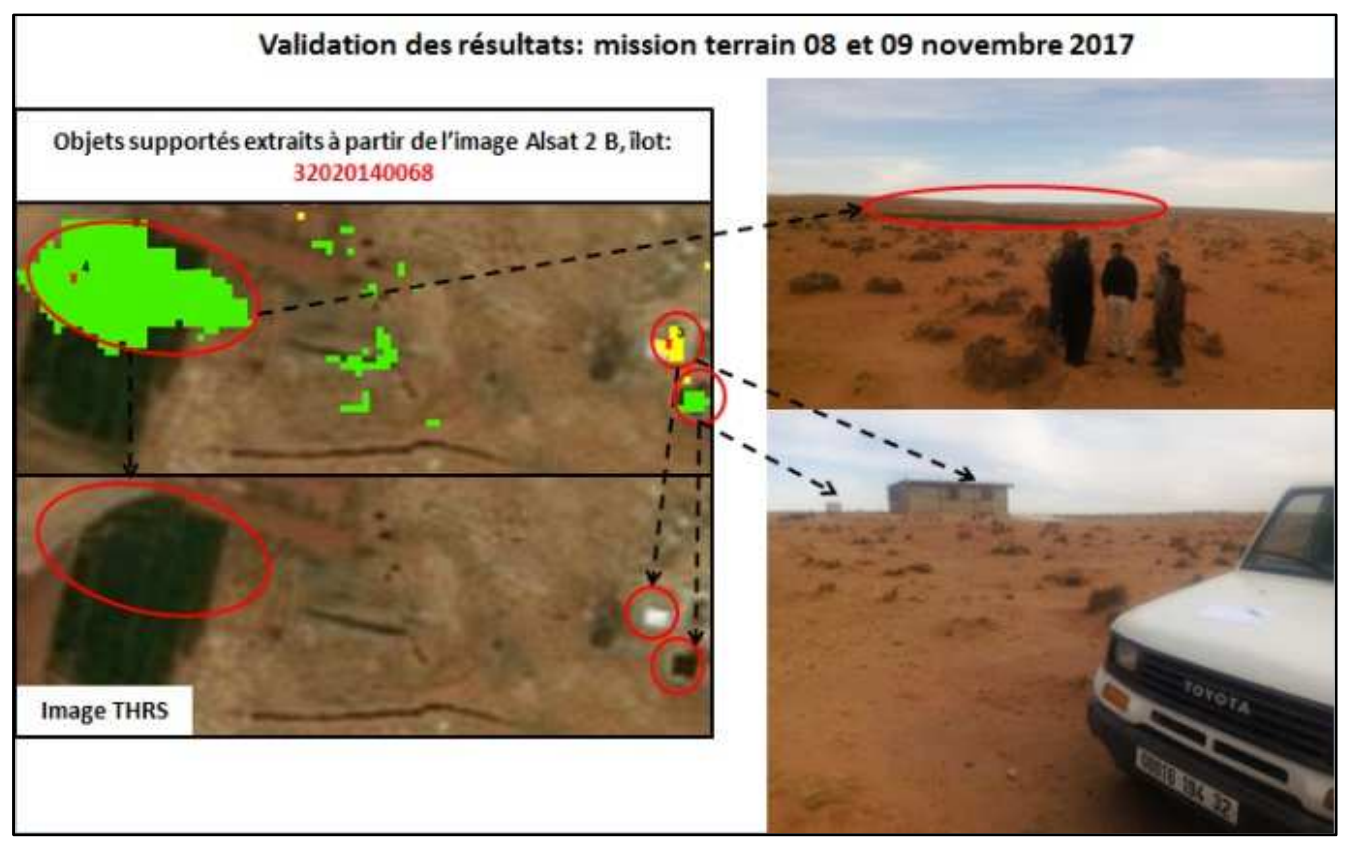

Figure 26 : Photos de terrains prises dans la commune de Rogassa (validation des extractions, coordonnées du centre de la parcelle $x=307000 m, y=3736000$, bâti en jaune $x=307244 m, y=3736145$ ).

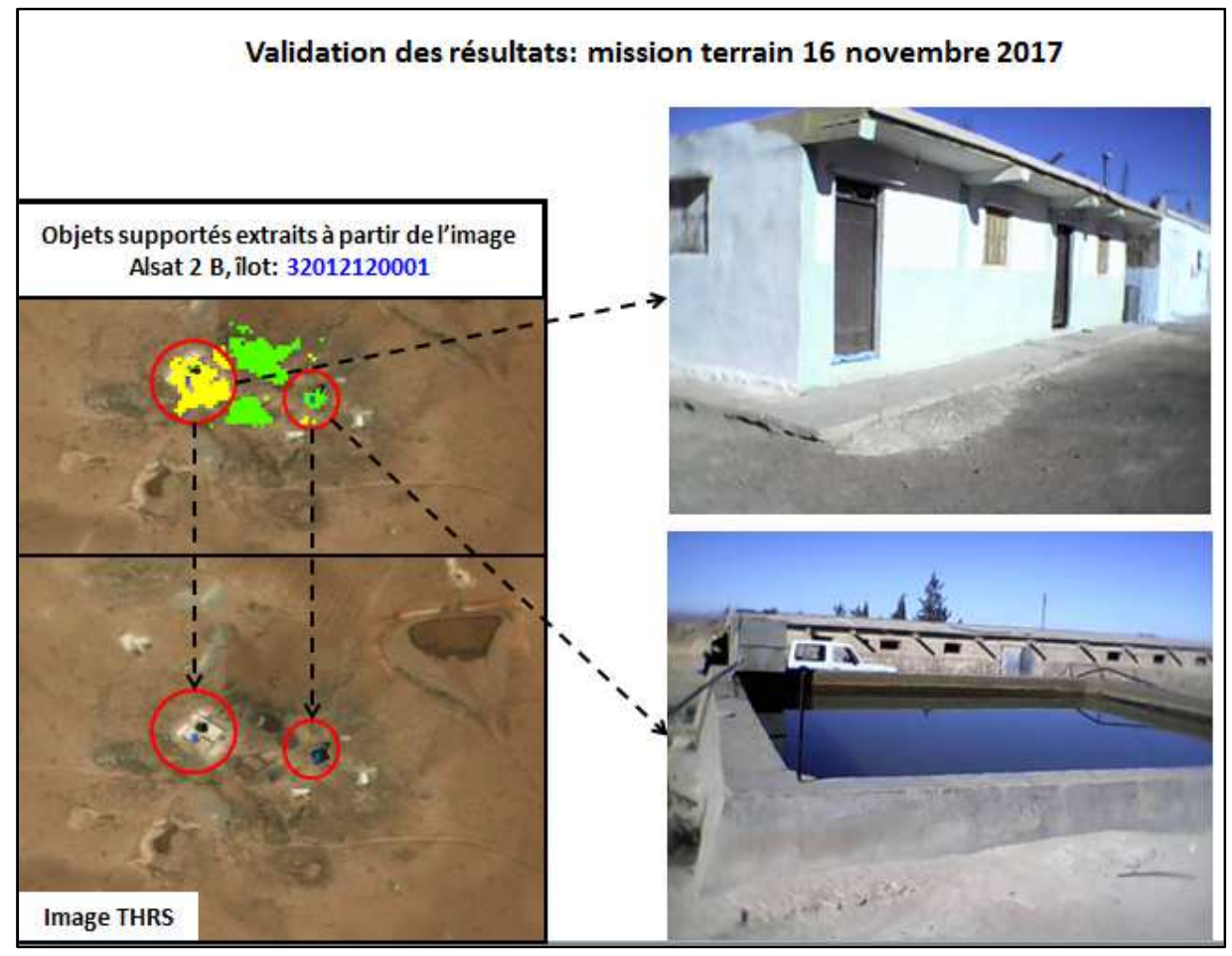

Figure 27 : Photos de terrains prises dans la commune d'El Bayadh (validation des extractions, Coordonnées du centre $x=312964 m, y=3734774 m)$.

\subsection{Conclusion}

La méthodologie suivie dans le cadre de ce travail repose sur le principe que chacun des classifieurs testés livre des informations certaines et d'autres en confusion (A. Masse, 2013), et sur la constitution d'une base de connaissances structurée comme objectif à atteindre avec l'approche orientée objet.
Durant tous les traitements, le choix des échantillons était en adéquation avec la nomenclature Spot Théma définie à une résolution spatiale équivalente à l'image support d'étude. Ainsi la notion de zone à exclure a été redéfinie de manière à intégrer toute possibilité d'existence de propriété privée dans le territoire cadastré. 
L'approche suivie pour extraire les informations utiles comprend trois phases :

- Identification des échantillons,

- Extraction et amélioration,

- Une reconnaissance d'objets extraits à partir des images THRS Google-Earth avant validation par les sorties sur terrain. Après enquête et délimitation (croquis de conservation ou document d'arpentage) et ensuite l'application des cas de mise à jour.

L'étude des propriétés spectrales nous a permis d'affiner la précision des choix des échantillons et de réduire la marge d'incertitude.

Contrairement à l'approche géométrique (qui a donné une valeur de 1/9000) l'analyse thématique (en utilisant la nomenclature de 13 objets sélectionnés à partir de Spot Théma) montre qu'une cartographie exhaustive à l'échelle $1 / 10000$ n'est pas possible alors qu'on peut assurer l'obtention du 1/25000 sans aucune difficulté.

\begin{tabular}{|c|c|c|}
\hline \multirow{2}{*}{ Nomenclature } & \multicolumn{2}{|c|}{ Echelles } \\
\cline { 2 - 3 } & $1 / 10000$ & $\mathbf{1 / 2 5 0 0 0}$ \\
\hline Culture annuelle et prairie & $\mathrm{x}$ & $\mathrm{x}$ \\
\hline Jardins familiaux & $\mathrm{x}$ & $\mathrm{x}$ \\
\hline Equipement de loisir & $\mathrm{x}$ & $\mathrm{x}$ \\
\hline Habitat discontinu collectif & - & $\mathrm{x}$ \\
\hline
\end{tabular}

\begin{tabular}{|c|c|c|}
\hline $\begin{array}{c}\text { Habitat discontinu } \\
\text { pavillonnaire }\end{array}$ & - & $\mathbf{x}$ \\
\hline Espace libre urbain & $\mathbf{x}$ & $\mathbf{x}$ \\
\hline $\begin{array}{c}\text { Coupe forestière et jeune } \\
\text { plantation }\end{array}$ & - & $\mathbf{x}$ \\
\hline Culture permanente & $\mathbf{x}$ & $\mathbf{x}$ \\
\hline Installation sportive & $\mathbf{x}$ & $\mathbf{x}$ \\
\hline Habitat isolé & - & $\mathbf{x}$ \\
\hline Grand équipement & $\mathbf{x}$ & $\mathbf{x}$ \\
\hline Tissu urbain continu & $\mathbf{x}$ & $\mathbf{x}$ \\
\hline Boisement linéaire & - & $\mathbf{x}$ \\
\hline
\end{tabular}

Tableau 4 : Exemple d'objets qui peuvent être cartographiés à partir de l'imagerie ALSAT2 aux échelles $1 / 10000$ et $1 / 20000$.

L'imagerie ALSAT2 peut répondre aux attentes du cadastre en zones steppiques en ce qui concerne l'identification des zones à exclure et même la cartographie à l'échelle $1 / 10000$ à $1 / 25000$. A une plus grande échelle il faut vérifier la possibilité d'intégrer des objets extraits directement dans les bases graphiques. Ainsi sur un échantillon de 17 objets de type bâti isolé en calculant les écarts en surface par rapport à la règle du $1 / 20^{\text {ème }}$ (expliquée ci-dessous) seuls 12 d'entre eux (cf. tableau $n^{\circ} 5$ ) ont des détails dont la superficie dépasse $200 \mathrm{~m}^{2}$ et peuvent être intégrés directement dans les îlots cadastraux.

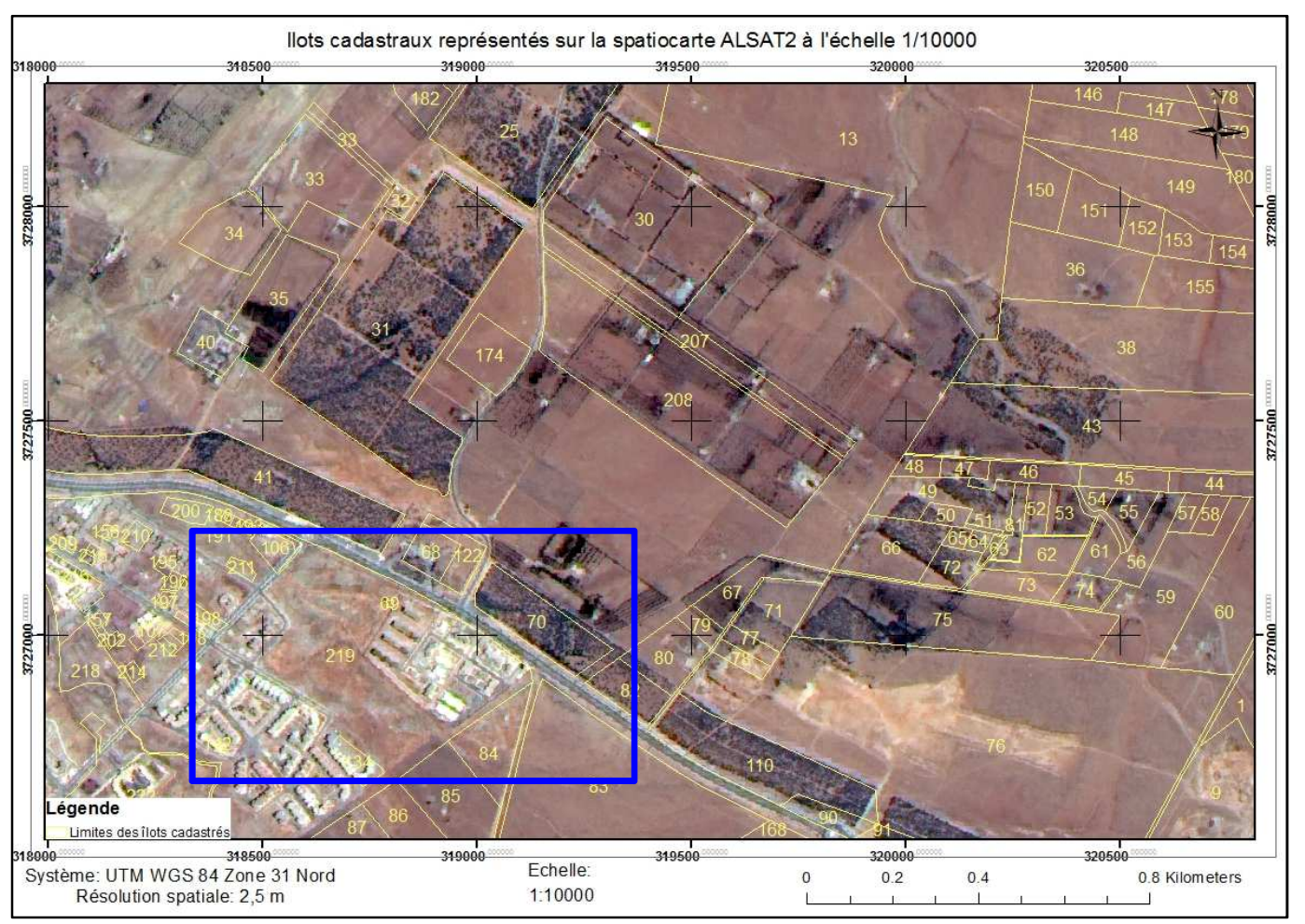

Figure 28 : Parfaite superposition des îlots cadastraux (en couleur jaune) sur la Spatiocarte à l'échelle 1/10000, la zone encadrée en bleu correspond à un exemple de la difficulté de l'image ALSAT2 pour la délimitation de l'habitat pavillonnaire discontinu cité en bleu sur le tableau 4 . 


\begin{tabular}{|c|c|c|c|}
\hline $\begin{array}{l}\text { Superficie ortho- } \\
\text { image (m2) }\end{array}$ & $\begin{array}{c}\text { Superficie } \\
\text { image } \\
\text { classifiée (m2) }\end{array}$ & $\begin{array}{l}\text { Ecart } \\
(\mathrm{m} 2)\end{array}$ & $\begin{array}{l}1 / 20 \text { de la } \\
\text { surface }\end{array}$ \\
\hline 444 & 418 & 26 & 22.2 \\
\hline 158 & 141 & 17 & 7.9 \\
\hline 291 & 296 & -5 & 14.55 \\
\hline 205 & 175 & 30 & 10.25 \\
\hline 278 & 319 & -41 & 13.9 \\
\hline 332 & 339 & -7 & 16.6 \\
\hline 99 & 82 & 17 & 4.95 \\
\hline 368 & 336 & 32 & 18.4 \\
\hline 134 & 100 & 34 & 6.7 \\
\hline 147 & 100 & 47 & 7.35 \\
\hline 113 & 95 & 18 & 5.65 \\
\hline 236 & 215 & 21 & 11.8 \\
\hline 278 & 221 & 57 & 13.9 \\
\hline 258 & 254 & 4 & 12.9 \\
\hline 543 & 526 & 17 & 27.15 \\
\hline 548 & 540 & 8 & 27.4 \\
\hline 553 & 536 & 17 & 27.65 \\
\hline
\end{tabular}

Tableau 5 : Contrôle de l'application de la règle du $1 / 20^{\mathrm{ème}}$ relative à la délivrance des livrets fonciers.
En Algérie la règle du 1/20ème doit être respectée pour la délivrance des livrets fonciers aux propriétaires ou les ayants droit. Elle consiste à une vérification de l'écart (qui ne doit pas dépasser 1/20) entre la superficie cadastrée et celle déclarée ou mentionnée sur l'acte.

Les images ALSAT2 permettent non seulement l'identification des parcelles et des objets existants (hangars, serres agricoles, bassins d'irrigation...) mais elles facilitent également l'accès sur le terrain aux propriétés et à leurs délimitations. Elles constituent un support terrain pour effectuer les opérations de maintenance des bases graphiques du cadastre plus facilement, avec une cartographie non exhaustive à l'échelle 1/10000 et complète à l'échelle 1/25000 (grands îlots appartenant à l'état).

Pour le cas des classes «bâti » la capacité de l'image est limitée à l'identification. Elle permet de localiser spatialement des segments indicatifs sur la possibilité de la présence d'une propriété omise (une confirmation est souhaitable avec l'imagerie THRS Google-Earth avant de sortir sur terrain).

La figure 29 donne l'exemple de l'îlot 68 (dont l'îlot primitif porte le numéro 05 , qui va faire l'objet d'une mise à jour après enquête foncière. II faut signaler que l'avantage de l'utilisation de l'imagerie ALSAT2 constitue une adaptation en matière du flux d'information entre le cadastre et la conservation foncière pour mieux gérer l'opération de délivrance des livrets fonciers.

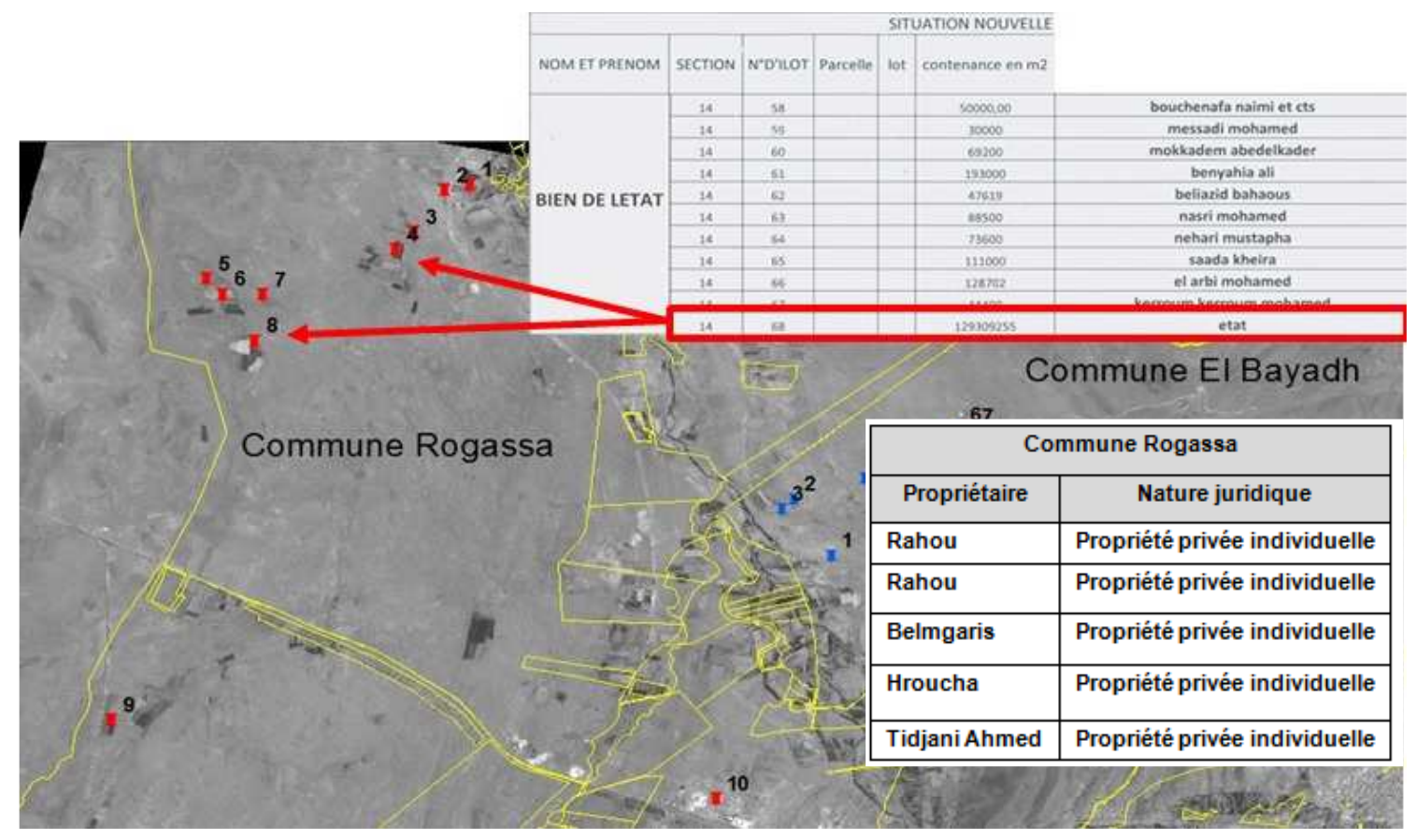

Figure 29 : Endroits de nos sorties sur terrain pour validation des résultats, cinq (05) propriétaires étaient présents sur le terrain et indiqués sur le tableau. Les propriétés enquêtées sont de nature juridique 'privées individuelles'. 
Enfin, la méthodologie développée durant ce travail a démontré l'intérêt de la télédétection opérationnelle dans le domaine du foncier à travers l'utilisation des orthoimages ALSAT2. Les résultats obtenus répondent aux exigences du cadastre steppique et saharien en Algérie. Ils permettent la maintenance de ses bases graphiques à des coûts compétitifs et dans les délais requis (comparativement à la photogrammétrie aérienne). II ressort de cette expérience que l'intégration de l'imagerie ALSAT2 dans le processus de maintenance engagé par l'Agence Nationale du Cadastre pourra faire l'objet d'un partenariat avec l'Agence Spatiale Algérienne.

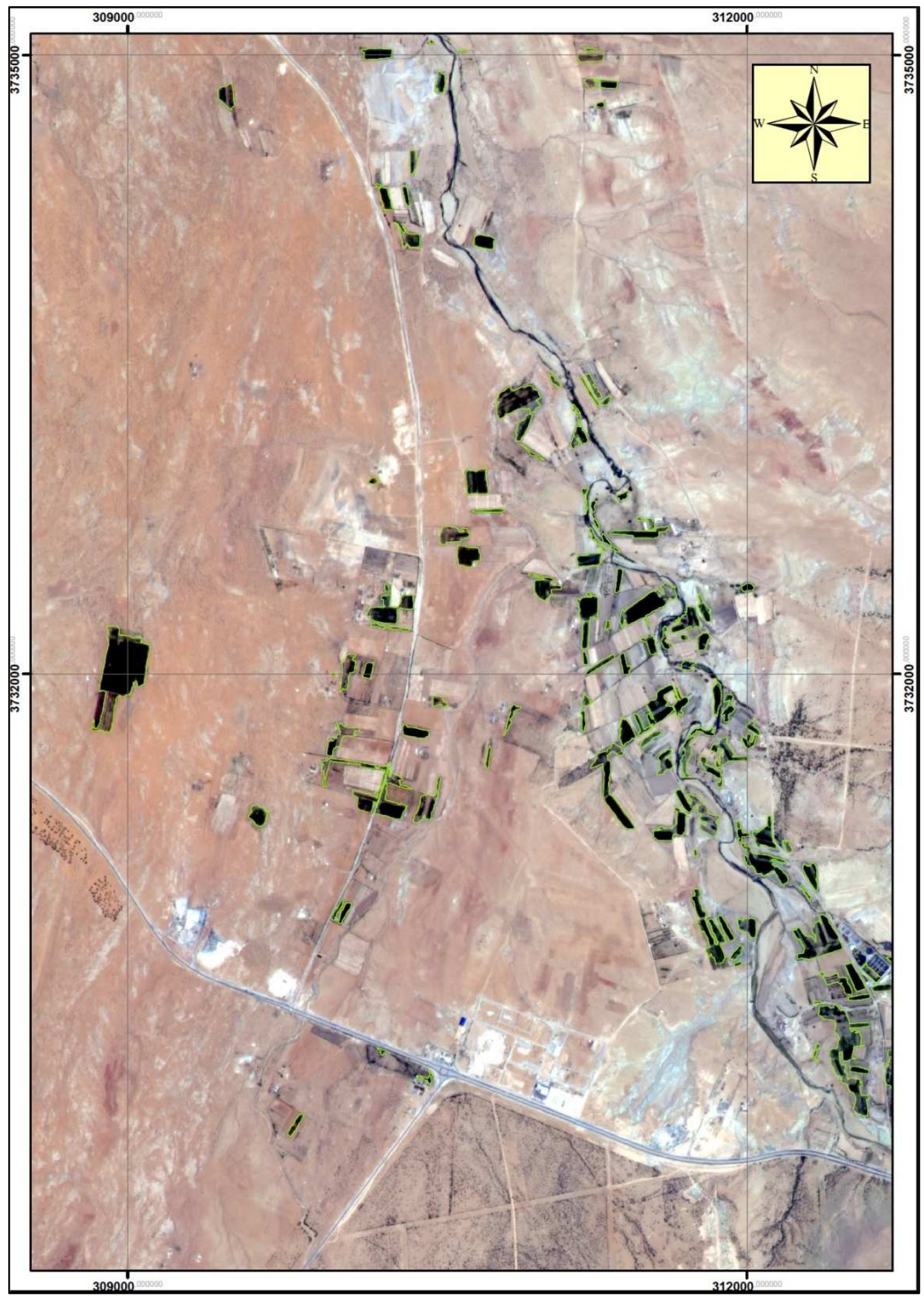

Figure 30 : Parcelles restituées automatiquement après extraction par approche orientée objet (à intégrer dans les bases graphiques cadastrales à l'échelle 1/10000, système UTM WGS84 Zone 31 N). 


\section{Références bibliographiques}

Ahmed Ali A, 2011 : « La législation foncière agricole en Algérie et les formes d'accès à la terre ". In: Elloumi M. (ed.), Jouve A.-M. (ed.), Napoléone $C$. (ed.), Paoli J.C. (ed.). Régulation foncière et protection des terres agricoles en Méditerranée. Mon tpellier: CIHEAM, 2011. p. 35-51 (Option s Méditerranéennes: Série $B$. Etudes et Recherches; n 66)

, http://om.ciheam.org/article.php?IDPDF=801372

Anne Puissant, 2003 : «Information géographique et images à très haute résolution utilité et applications en milieu urbain ». Géographie. Université Louis Pasteur - Strasbourg I, 2003. Français. tel00467474, https://tel.archives-ouvertes.fr/tel00467474. P 291

Antoine Masse, 2013: «Développement et automatisation de méthodes de classification à partir de séries temporelles d'images de télédétection - Application aux changements d'occupation des sols et à l'estimation du bilan carbone. Océan, Atmosphère. Université Paul Sabatier - Toulouse III. Français. tel-00921853v2

Astrium GEO Information Services, 2011: «SPOT THEMA Base de Données d'Occupation du Sol pour les Acteurs du Territoire ». GE0035-0313, P2 que ce que Spot Théma?

Caitlin Dempsey, 2000: «GIS Learning National Map Accuracy Standards (NMAS)". https://www.gislounge.com/national-mapaccuracy-standards-nmas/.

D.Holland, 2006 : « Updating topographic mapping in Great Britain using imagery from high-resolution satellite sensors ", ISPRS Journal of Photogrammetry and Remote Sensing 60(3):212 223,DOI: 10.1016/j.isprsjprs.2006.02.002.

F. G. D. Committee, 1998: « Geospatial Positioning Accuracy Standards, Part 3: National Standard for Spatial Data Accuracy». Subcomm. Base Cartogr. Data.

Françoise Gourmelon, 2005: «Potentialités de l'imagerie satellitaire spot 5 pour la cartographie de la végétation ». Revue CyberGeo, https://doi.org/10.4000/cybergeo.3027.P 36.

\section{LEGENDES EN ANGLAIS}

Figure 1: Location of the steppe territory on the map of Algeria ( $14 \%$ of the national territory).

Figure 2: AISAT2 ortho-image draped over the DTM of the city of El Bayadh (average altitude of $1313 \mathrm{~m}$ ).

Figure 3: Example of a segment composed of two ALSAT2 images.
Herbert J. Kramer, 2002: "Observation of the Earth and Its Environment: Survey of Missions and Sensors", (Springer Verlag). https://directory.eoportal.org/web/eoportal/satellite -missions/a/alsat-2.

Lhomme, Stéphane, 2005 : « Identification du bâti à partir d'images satellitaires à très hautes résolutions spatiales ", Thèse en Géomatique appliquée, université de Sherbrooke. http://hdl.handle.net/11143/5824.

Loi n`90-25 du 18/11/1990, modifiée et complétée, portant orientation foncière. http://madrp.gov.dz/wpcontent/uploads/2019/01/L oi $90251811 \quad 1990$ orientation fonciere.pdf

M. Corlazzoli and O. L. Fernandez, 2004: «SPOT 5 Cadastral validation project in Izabal, Guatemala». Int. Arch. Photogramm. Remote Sens. Spat. Inf. Sci., vol. 35, no. Part 1, pp. 291-296.

M. Ettarid, f. Degaichia, 2004: «Potentiel cartographique de l'imagerie Ikonos " Commission IV,

WGIV/7.https://www.isprs.org/proceedings/xxxv/co ngress/comm4/papers/519.pdf.

P. A. Handbook, 1999: « Using the National Standards for Spatial Data Accuracy to measure and report geographic data quality». Minn. Plan. Land Manag. Inf. Cent.

P. Jayaprasad et al, 2006: « Updation and metrology of cadastre and village boundary using high resolution satellite data». Int. Arch. Photogramm. REMOTE Sens., vol. 36.

Riad BENSOUIAH, 2003: "La lutte contre la désertification dans la steppe algérienne: les raisons de l'échec de la politique environnementale »Communication présentée aux 15èmes Journées de la Société d'Ecologie Humaine organisée à Marseille (France) sous le thème: " Du Nord au Sud: le recours à l'environnement, le retour des paysans ?", $P 9$ et 10.

Figure 4: Zoom in on the blue frame $d$ area on the ALSAT2 segment (Figure 3).

Figure 5: Spatiomap from an ALSAT2 multispectral image of the city of El Bayadh.

Figure 6: Spatiomap of a panchromatic ALSAT2 image of El Bayadh airport (UTM system WGS 84 Zone 31 North, $x \min =321000$, $y \min =3732000, x \max =325500$, $y \max =3734000 \mathrm{~m}$ ) 
Figure 7: Spatiomap of the cadastral survey area $(x \min =307000, \quad y \min =3731000, \quad x \max =314000$, $y \max =3735000 \mathrm{~m})$.

Figure 8: Cadastral references of the selected sites (UTM system WGS 84 Zone 31 North, xcentroid $=311398$, ycentroid $=3732773 \mathrm{~m}$ ).

Figure 9: Spatial distribution on the ALSAT2 image of samples selected according to the nomenclature of Spot-Théma objects (UTM system WGS 84 Zone 31 North, $x \min =301500, y \min =3720000, x \max =323000$, $y \max =3740000 \mathrm{~m})$.

Figure 10: Examples of spectral properties of some samples selected according to the nomenclature of Spot-Théma objects (their identification with enlargement from the ALSAT2 image and on VHSR images type Google-Earth). The blue circles correspond to built objects that cannot be considered as isolated because of their proximity to roads or their positions within parcels (easily identifiable).

Figure 11: Methodological approach developed for the identification of areas of interest (blocks of built or unbuilt property) as part of the maintenance of the steppe cadaster.

Figure 12: Example of thematic confusions (pixels in yellow) due to single-band extraction (xcentroid=312623, ycentroid=3729383m).

Figure 13: Example of thematic confusions (pixels in yellow) due to extraction by applying the K-Means algorithm (xcentroid=312819m, ycentroid=3729944m).

Figure 14: Example of thematic confusions (pixels in yellow) due to extraction by applying the ISODATA algorithm (xcentroid=312819m, ycentroid $=3729944 \mathrm{~m}$ ).

Figure 15: Example of thematic confusions (pixels in red) due to extraction by applying maximum likelihood supervised classification. (xmin $=301600, \quad y \min =$ $3719520, x \max =322926, y \max =3735924 \mathrm{~m})$.

Figure 16: Statistics of the number of built objects extracted by different classification algorithms.

Figure 17: Comparison of the extraction statistics of the vegetation object.

Figure 18: Enlargement of the ALSAT2 Spatiomap at 1:2500 scale on which are circled in blue omitted built objects and parcels that belong to block 3 and 43 cadastred in the name of the state.

Figure 19: Examples of extracted plots A (scattered plots), B (collective farms EAC), C (peri-urban vegetation). ycentroide $=3728632 \mathrm{~m}$ )

Figure 20: Zoom on class A (in green color) equivalent extraction level at $1 / 10000$ scale (xcentroide $=307120 \mathrm{~m}$, ycentroide $=3732058 \mathrm{~m}$ ).
Figure 21: Zoom on class B (in green color) equivalent extraction level at $1 / 10000$ scale (xcentroide $=312164 \mathrm{~m}$, ycentroide $=3732177 \mathrm{~m})$.

Figure 22: Examples of the identified building (in the form of grouping of industrial type or other, it is the class 01 , or isolated class 02) (xcentroide $=315500 \mathrm{~m}$, ycentroide $=3724045 \mathrm{~m}$ )

Figure 23: Examples of isolated buildings extracted class 2 on figure 22 (objects framed in blue on figure 10). (xcentroide $=316200 \mathrm{~m}$, ycentroide $=3722751 \mathrm{~m}$ ).

Figure 24: Degradation of the visual quality of the 1:2500 scale orthoimage (different zoom level than in Figure 18)

Figure 25: Identification of building and road segments (in blue) from the ALSAT2 image displayed at 1:2500 scale.

Figure 26: Photos of land taken in the municipality of Rogassa (validation of extractions, coordinates of the center of the parcel $x=307000 m, y=3736000$, frame in yellow $x=307244 \mathrm{~m}, \mathrm{y}=3736145$ ).

Figure 27: Photos of land taken in the commune of $\mathrm{El}$ Bayadh (validation of extractions, center coordinates $x=312964 m, y=3734774 m)$.

Figure 28: Perfect superposition of the cadastral property (in yellow color) on the Spatiomap at 1/10000 scale, the area framed in blue corresponds to an example of the difficulty of the ALSAT2 image for the delimitation of the discontinuous suburban habitat cited in blue on Table 4.

Figure 29: Locations of our field trips for validation of results, five (05) owners were present in the field and indicated on the table. The properties surveyed are of a 'private individual' legal nature.

Figure 30: Plots of land automatically restored after extraction by object-oriented approach (to be integrated into the cadastral graphic databases at a scale of 1/10000, UTM WGS84 Zone $31 \mathrm{~N}$ system).

Table 1: Example of ALSAT2 image metadata

Table 2: GPS and ALSAT2 Orthoimage point coordinates used for scaling.

Table 3: Determination of the map scale of the ALSAT2 image used for the study.

Table 4: Example of objects that can be mapped from ALSAT2 imagery at $1 / 10000$ and $1 / 20000$ scales.

Table 5: Control of the application of the 1/20 meter rule for the issuance of land registers.

Table A1: Statistics of the extraction of the built object by single band analysis

Table A2: Statistics of the extraction of the built object by Isodata algorithm

Table A3: Statistics of the extraction of the built object by K-Means algorithm

Table A4: Statistics of the extraction of the built object by supervised classification (maximum likelihood)

Table A6: Summary of uncertainties 


\section{Annexe}

\begin{tabular}{|c|c|c|c|c|c|c|c|}
\hline \multicolumn{8}{|c|}{ Extraction supervisée Mono bande : objet bâti isolé } \\
\hline Commune & îlot & $\begin{array}{l}\text { superficie } \\
\text { m2 }\end{array}$ & $\begin{array}{l}\text { Extraction } \\
\text { brute }\end{array}$ & $\begin{array}{c}\text { Après } \\
\text { nettoyage }\end{array}$ & $\begin{array}{c}\text { Confusion avec } \\
\text { objet route }\end{array}$ & $\begin{array}{c}\text { Confusion } \\
\text { avec d'autres } \\
\text { objets }\end{array}$ & $\begin{array}{l}\text { Objets } \\
\text { validés }\end{array}$ \\
\hline \multirow{13}{*}{ El Bayadh } & 2 & 353347 & 113 & 16 & 0 & 16 & 0 \\
\hline & 18 & 142945 & 116 & 11 & 0 & 9 & 2 \\
\hline & 4 & 35398 & 23 & 1 & 0 & 0 & 1 \\
\hline & 5 & 446761 & 52 & 10 & 0 & 6 & 4 \\
\hline & 6 & 446761 & 41 & 7 & 0 & 6 & 1 \\
\hline & 7 & 2865137 & 353 & 21 & 0 & 13 & 8 \\
\hline & 12 & 148160 & 91 & 4 & 0 & 2 & 2 \\
\hline & 37 & 538384 & 81 & 10 & 8 & 0 & 2 \\
\hline & 39 & 1523879 & 112 & 35 & 35 & 0 & 0 \\
\hline & 40 & 209450 & 53 & 9 & 0 & 9 & 0 \\
\hline & 45 & 82432 & 1 & 1 & 0 & 0 & 1 \\
\hline & 54 & 88663 & 23 & 2 & 0 & 1 & 1 \\
\hline & 241 & 198089 & 31 & 3 & 0 & 3 & 0 \\
\hline \multirow{4}{*}{ Rogassa } & 5 & 129395443 & 98 & 14 & 0 & 13 & 1 \\
\hline & 6 & 19034257 & 614 & 62 & 42 & 8 & 12 \\
\hline & 65 & 109454 & 36 & 5 & 0 & 5 & 0 \\
\hline & 68 & 125577517 & 2405 & 426 & 323 & 37 & 66 \\
\hline Total & & & 4243 & & & & 101 \\
\hline
\end{tabular}

Tableau A1 : Statistiques de l'extraction de l'objet bâti par analyse mono bande

\begin{tabular}{|c|c|c|c|c|c|c|c|}
\hline \multicolumn{8}{|c|}{ Extraction non supervisée IsoData multi bandes: objet bâti isolé } \\
\hline Commune & îlot & $\begin{array}{l}\text { Surface } \\
\text { m2 }\end{array}$ & $\begin{array}{l}\text { Extraction } \\
\text { brute }\end{array}$ & $\begin{array}{c}\text { Après } \\
\text { nettoyage }\end{array}$ & $\begin{array}{c}\text { Confusion } \\
\text { avec objet } \\
\text { route }\end{array}$ & $\begin{array}{l}\text { Confusion avec } \\
\text { d'autres objets }\end{array}$ & Objets validés \\
\hline \multirow{9}{*}{$\begin{array}{c}\text { El } \\
\text { Bayadh }\end{array}$} & 1 & 12511088 & 744 & 216 & 34 & 175 & 7 \\
\hline & 2 & 353347 & 88 & 21 & 0 & 18 & 3 \\
\hline & 3 & 445024 & 53 & 16 & 0 & 11 & 5 \\
\hline & 4 & 35398 & 2 & 1 & 0 & 0 & 1 \\
\hline & 12 & 148160 & 69 & 16 & 0 & 11 & 5 \\
\hline & 18 & 142945 & 80 & 23 & 0 & 23 & 0 \\
\hline & 37 & 538384 & 37 & 8 & 3 & 2 & 3 \\
\hline & 48 & 159236 & 5 & 2 & 0 & 1 & 1 \\
\hline & 54 & 88663 & 10 & 5 & 0 & 1 & 4 \\
\hline \multirow{3}{*}{ Rogassa } & 6 & 19034257 & 569 & 129 & 40 & 71 & 18 \\
\hline & 5 & 129395443 & 68 & 18 & 0 & 16 & 2 \\
\hline & 68 & 125577517 & 2650 & 652 & 76 & 541 & 35 \\
\hline Total & & & 4375 & & & & 84 \\
\hline
\end{tabular}

Tableau A2 : Statistiques de l'extraction de l'objet bâti par algorithme Isodata 


\begin{tabular}{|c|c|c|c|c|c|c|c|}
\hline \multicolumn{8}{|c|}{ Extraction non supervisée K-Means multi bandes: objet bâti isolé } \\
\hline Commune & Ilot & $\begin{array}{l}\text { Surface } \\
\text { m2 }\end{array}$ & $\begin{array}{l}\text { Extraction } \\
\text { brute }\end{array}$ & $\begin{array}{c}\text { Après } \\
\text { nettoyage }\end{array}$ & $\begin{array}{l}\text { Confusion } \\
\text { avec objet } \\
\text { route }\end{array}$ & $\begin{array}{l}\text { Confusion avec } \\
\text { d'autres objets }\end{array}$ & $\begin{array}{l}\text { Objets } \\
\text { validés }\end{array}$ \\
\hline \multirow{13}{*}{ El Bayadh } & 2 & 353347 & 37 & 8 & 0 & 8 & 0 \\
\hline & 18 & 142945 & 55 & 8 & 0 & 8 & 0 \\
\hline & 4 & 35398 & 12 & 2 & 0 & 0 & 2 \\
\hline & 5 & 446761 & 5 & 1 & 0 & 1 & 0 \\
\hline & 7 & 2865137 & 405 & 97 & 8 & 61 & 28 \\
\hline & 12 & 148160 & 78 & 18 & 11 & 1 & 6 \\
\hline & 37 & 538384 & 119 & 7 & 1 & 5 & 1 \\
\hline & 39 & 1523879 & 44 & 8 & 0 & 8 & 0 \\
\hline & 40 & 209450 & 22 & 5 & 0 & 5 & 0 \\
\hline & 54 & 88663 & 34 & 4 & 0 & 4 & 0 \\
\hline & 241 & 198089 & 59 & 7 & 0 & 7 & 0 \\
\hline & 1 & 12511088 & 1078 & 185 & 0 & 164 & 21 \\
\hline & 3 & 445024 & 35 & 6 & 0 & 4 & 2 \\
\hline \multirow{3}{*}{ Rogassa } & 6 & 19034257 & 331 & 47 & 43 & 2 & 2 \\
\hline & 65 & 109454 & 23 & 5 & 0 & 4 & 1 \\
\hline & 68 & 125577517 & 1487 & 74 & 12 & 53 & 9 \\
\hline Total & & & 3824 & & & & 72 \\
\hline
\end{tabular}

Tableau A3 : Statistiques de l'extraction de l'objet bâti par algorithme K-Means

\begin{tabular}{|c|c|c|c|c|c|c|c|}
\hline \multicolumn{8}{|c|}{ Extraction supervisée Multi bandes : objet bâti isolé } \\
\hline Commune & îlot & Surface $\mathrm{m} 2$ & Extraction brute & Après nettoyage & $\begin{array}{c}\text { Confusion avec } \\
\text { objet route }\end{array}$ & $\begin{array}{l}\text { Confusion avec } \\
\text { d'autres objets }\end{array}$ & Objets validés \\
\hline \multirow{13}{*}{$\begin{array}{c}\text { El } \\
\text { Bayadh }\end{array}$} & 1 & 12511088 & 3496 & 700 & 75 & 624 & 1 \\
\hline & 2 & 353347 & 219 & 42 & 0 & 38 & 4 \\
\hline & 18 & 142945 & 55 & 8 & 0 & 8 & 0 \\
\hline & 4 & 35398 & 33 & 5 & 0 & 3 & 2 \\
\hline & 5 & 446761 & 155 & 29 & 0 & 21 & 8 \\
\hline & 6 & 446761 & 47 & 11 & 0 & 8 & 3 \\
\hline & 7 & 2865137 & 588 & 120 & 0 & 106 & 14 \\
\hline & 12 & 142945 & 141 & 38 & 0 & 32 & 6 \\
\hline & 37 & 538384 & 122 & 119 & 0 & 116 & 3 \\
\hline & 54 & 88663 & 58 & 20 & 0 & 15 & 5 \\
\hline & 241 & 198089 & 98 & 19 & 0 & 18 & 1 \\
\hline & 3 & 445024 & 180 & 33 & 0 & 30 & 3 \\
\hline & 48 & 159236 & 34 & 6 & 0 & 2 & 4 \\
\hline \multirow{3}{*}{ Rogassa } & 6 & 19034257 & 7803 & 1532 & 253 & 1251 & 28 \\
\hline & 5 & 129395443 & 358 & 100 & 6 & 89 & 5 \\
\hline & 68 & 125577517 & 14138 & 2723 & 393 & 2277 & 53 \\
\hline Total & & & 27525 & & & & 140 \\
\hline
\end{tabular}

Tableau A4 : Statistiques de l'extraction de l'objet bâti par classification supervisée (maximum de vraisemblance) 


\begin{tabular}{|c|c|c|c|c|c|c|}
\hline \multicolumn{7}{|c|}{ Extraction du bâti par règles d'apprentissage (sans nettoyage) } \\
\hline Commune & îlot & Superficie & $\begin{array}{c}\text { Extraction } \\
\text { brute }\end{array}$ & $\begin{array}{c}\text { Avec objet } \\
\text { route }\end{array}$ & $\begin{array}{c}\text { Avec d'autres } \\
\text { objets }\end{array}$ & $\begin{array}{l}\text { Objets } \\
\text { validés }\end{array}$ \\
\hline \multirow{7}{*}{$\begin{array}{c}\text { El } \\
\text { Bayadh }\end{array}$} & 1 & 12511087 & 42 & 10 & 24 & 8 \\
\hline & 2 & 353346 & 2 & 1 & 0 & 1 \\
\hline & 3 & 445023 & 4 & 0 & 1 & 3 \\
\hline & 12 & 148159 & 4 & 0 & 1 & 3 \\
\hline & 18 & 142945 & 2 & 0 & 0 & 2 \\
\hline & 45 & 82431 & 1 & 0 & 0 & 1 \\
\hline & 5 & 446760 & 1 & 0 & 0 & 1 \\
\hline \multirow{4}{*}{ Rogassa } & 6 & 19034257 & 29 & 5 & 9 & 15 \\
\hline & 5 & 129395443 & 3 & 0 & 2 & 1 \\
\hline & 68 & 125577517 & 155 & 26 & 85 & 44 \\
\hline & 64 & 72575 & 2 & 0 & 1 & 1 \\
\hline Total & & & 245 & & & 80 \\
\hline
\end{tabular}

Tableau A5 : statistiques de l'extraction de l'objet bâti par approche orientée objet

\begin{tabular}{|c|c|c|c|c|}
\hline \multicolumn{2}{|c|}{ Classification } & Extraction brute & Information certaine en\% & Information incertaine en $\%$ \\
\hline \multicolumn{2}{|c|}{$\begin{array}{c}\text { Segmentation mono } \\
\text { bandes }\end{array}$} & 637 & 15.85 & 84.14 \\
\hline \multirow{2}{*}{$\begin{array}{c}\text { Non } \\
\text { supervisée }\end{array}$} & ISODATA & 1107 & 14.93 & 92.41 \\
\hline & K-MEANS & 482 & 7.58 & 85.06 \\
\hline \multicolumn{2}{|c|}{$\begin{array}{c}\text { Supervisée Maximum de } \\
\text { vraisemblance }\end{array}$} & 5505 & 2.543 & 97.45 \\
\hline \multicolumn{2}{|c|}{ Orientée objet } & 245 & 33.06 & 66.94 \\
\hline
\end{tabular}

Tableau A6 : récapitulatif des incertitudes

NB : Suite au taux d'incertitude de 60\% (extraction brute) de l'approche orientée objet pour le cas du bâti isolé, une confirmation de l'identification à partir des images Google-Earth est nécessaire avant d'amorcer les contrôles sur terrain. 